\title{
The Bright Homunculus in our Head: Individual Differences in Intuitive Sensitivity to Logical Validity
}

\author{
Omid Ghasemi ${ }^{1}$, Simon Handley ${ }^{1}$, Stephanie Howarth ${ }^{1}$, \\ 1 Department of Cognitive Science, Macquarie University, Sydney, Australia \\ This manuscript is published in Quarterly Journal of Experimental Psychology \\ https://doi.org/10.1177/17470218211044691
}

Corresponding author:

Omid Ghasemi (Macquarie University, Sydney, NSW, 2109, AUSTRALIA)

Email: omidreza.ghasemi@hdr.mq.edu.au

This research was supported by the International Macquarie University Research Excellence Scholarship (iMQRES, Allocation No. 2018459/20191525) Which was awarded to O. G. All of the materials and data are accessible via the Open Science Framework website (https://osf.io/5dvcs/). 


\begin{abstract}
Classic dual process theories of human reasoning attribute explicit reasoning to effortful, deliberative thinking. According to these models, intuitive processes lack any access to the formal rules of logic and probability and hence rely exclusively on superficial problem features to determine a response. However, in recent years, researchers have demonstrated that reasoners are able to solve simple logical or probabilistic problems relatively automatically, a capability which has been called 'logical intuition'. In four experiments, we instructed participants to judge the validity (Experiments 1 and 4), likeability (Experiments 1,2, and 3) and physical brightness (Experiments 2, 3, and 4) of the conclusion to several reasoning problems. Brightness judgments were made by evaluating the font shade brightness of the argument's conclusion. Participants were also asked to complete a range of individual differences measures, drawing on cognitive ability and cognitive style, in order to evaluate the extent to which 'logical intuitions' were linked to measures of deliberative reasoning. The results showed that participants judged the conclusion of logically valid statements to be more valid, more likable and more physically bright than invalid statements. Participants with higher cognitive ability and unlimited processing time showed greater effects of logical validity in their liking judgments (varied across experiments). However, these effects were absent in the brightness tasks, suggesting that logic effects observed under instructions to judge conclusion brightness are a purer measure of 'logical intuition'. We discuss the implications of our findings for recent dual process theories of human reasoning.
\end{abstract}

Keywords: Reasoning; Dual process theory; Logical intuition; Cognitive ability; Individual differences 


\section{Introduction}

The idea of two distinct processes, intuition and reasoning, in one mind is a dominant view within cognitive and social psychology. A classic example is the dual process theory of reasoning and decision making. According to this account, two qualitatively distinct processes are involved in judgment and reasoning: Type 1 (T1) or intuitive processes with automaticity as their defining feature and Type 2 (T2) or deliberative processes with dependence on working memory and cognitive decoupling from irrelevant features (i.e., believability of a problem) as their necessary features (De Neys, 2014; Evans \& Stanovich, 2013; Handley \& Trippas, 2015; Kahneman \& Frederick, 2005; Sloman, 2012; but see, Melnikoff \& Bargh, 2018). Although theorists commonly reject attributing biased thinking to T1 processes and normative responding to T2 thinking (Evans, 2017; Evans \& Stanovich, 2013; Gigerenzer \& Gaissmaier, 2010; Pennycook, De Neys, Evans, Stanovich, \& Thompson, 2018), the failure to engage in deliberative thinking has been assumed as the main factor explaining biased responding in reasoning and decision-making contexts (Epstein, 1994; Kahneman \& Frederick, 2005; Morewedge \& Kahneman, 2010). Consistent with this account, intuitive thinking has been described as arising from an unintelligent and frenetic homunculus in the brain who insists on the wrong answer regardless of its evident incorrectness (Gould, 1989).

According to this view, the accessibility of rules, such as the rules of probability or deductive logic, are exclusive to $\mathrm{T} 2$ processes whereas, $\mathrm{T} 1$ processes rely primarily on superficial cues such as the matching of different elements of the problem (e.g., matching bias), stereotypical characteristics (e.g., base-rate neglect), believability and acceptability of the content (e.g., belief bias and myside bias), and the co-occurrence information and associative representations (e.g., conjunction fallacy; Evans \& Stanovich, 2013; Klauer, Musch, \& Naumer, 2000; Sloman, 1996; Tversky \& Kahneman, 1983). The direct correspondence between cognitive processes and types of response has been challenged in a number of recent studies, even by those who argue for such a correspondence in the first place. In the 
following sections, we review recent evidence that is consistent with the existence of 'logical intuitions' and individual differences in such a capability.

\subsection{Logical Intuitions}

A number of recent experimental findings suggest that $\mathrm{T} 1$ thinking can produce the correct normative answer without any engagement of T2 processes (For a review, see, De Neys \& Pennycook, 2019). In fact, it is not necessary for reasoners to delay their responses until the involvement of the corrective T2 processes, because they often reach the correct answer by using their fast $\mathrm{T} 1$ processes. For example, across seven studies, Bago and De Neys (2019b) used a two-response paradigm in which participants were presented with a problem twice; once under limited time and cognitive load and once with no time pressure or load. These researchers demonstrated that for participants who answered reasoning problems correctly, those answers were mainly generated under limited time and limited cognitive capacity. In other words, correct responses were mainly raised from intuitive T1 thinking, rather than corrective T2 deliberative thinking. Similarly, Newman, Gibb, and Thompson (2017) found that reasoners can make rule-based inferences even when they are required to answer in a limited time.

In typical reasoning studies, participants are asked to judge whether a conclusion necessarily follows from the premises (see, for example, Evans et al., 2009; Newstead et al., 1992). Instead, Handley and colleagues used a different instructional manipulation, in order to test the direct correspondence hypothesis of the traditional dual process theory, which asked participants to judge conflict and no-conflict problems ${ }^{1}$ based on either the logical validity or the believability of the presented conclusion. If there is a direct correspondence between logic and belief judgments on the one hand, and $\mathrm{T} 1$ and $\mathrm{T} 2$ processes on the other hand (i.e., belief corresponds to $\mathrm{T} 1$ thinking and logic corresponds to T2 thinking), reasoners should find logic judgments more difficult since this judgment demands cognitive resources compared to the belief judgment. Contrary to this prediction, the findings

\footnotetext{
${ }^{1}$ A conflict problem is a kind of problem in which the intuitive response is at odds with the response cued by deliberative processes (e.g., All flowers need water; Roses need water; Roses are flowers). A no-conflict problem is one in which its validity and believability cue a similar response (e.g., All flowers need water; Roses are flowers; Roses need water).
} 
revealed that reasoners under logic instructions had greater accuracy and faster latency. More importantly, logic interfered with belief (the conflict between validity and believability of the problem had a pronounced effect under belief instruction), while belief did not interfere with logic to the same extent. These findings have been replicated across different reasoning tasks (Howarth, Handley, \& Walsh, 2016, 2018; Pennycook, Trippas, Handley, \& Thompson, 2014) with different levels of complexity (Trippas, Thompson, \& Handley, 2017) and experimental designs (Handley, Newstead, \& Trippas, 2011). These theorists suggest that, at least on simple problems, logical inferences depend on automatic processes and are more accessible than belief evaluations. These findings indicate that reasoners can make inferences based on logical and probabilistic rules relatively fast (via T1 thinking) whilst making belief-based inferences relatively slowly (Howarth \& Handley, 2016; Trippas \& Handley, 2018).

Additional evidence for the presence of logical intuitions comes from conflict detection studies. In these studies, participants are presented with conflict and no-conflict versions of different reasoning tasks. When responding to conflict problems compared to no-conflict problems, participants, irrespective of whether their final response is accurate or biased, show an increase in response time (De Neys \& Glumicic, 2008; Frey, Johnson, \& De Neys, 2017), an increase in skin conductance responses (De Neys, Moyens, \& Vansteenwegen, 2010), an increased tendency to re-view critical elements of the problem (De Neys \& Glumicic, 2008), a decrease in the confidence level (De Neys, Cromheeke, \& Osman, 2011; Thompson \& Johnson, 2014), and an increase in the activation of anterior cingulate cortex (De Neys, Vartanian, \& Goel, 2008) and centro-parietal N2 and frontal P3 (Bago et al., 2018), regions which have been attributed mainly to the conflict detection sensitivity. Detecting the conflict, which generally happens implicitly, indicates that reasoners can automatically evaluate the consistency of their initial responses with the correct normative responses without engaging in T2 processes (Bago $\&$ De Neys, 2017a, 2017b; De Neys, 2014). Individuals may have no explicit clue what the exact correct answer is, although, they still appear to have an implicit knowledge of the correct logical response. 
An important question concerns the mechanisms that underpin intuitive sensitivity to logical structure. According to the fluency-affect intuition model (Topolinski, 2011, 2017), coherency leads to higher levels of fluency, which in turn, due to hedonically marked experience, results in positive affect. This affect, at the later stages of the process, may be used as a cue for intuitive judgment. Morsanyi and Handley (2012) applied the fluency-affect paradigm to the reasoning domain, arguing that logically valid arguments would be processed more fluently than invalid arguments as participants would more readily integrate the premises and conclusions into a coherent model. In order to test this hypothesis, they asked participants to rate how much they liked the presented conclusion of valid and invalid argument forms. In 4 experiments, they showed that participants liked and endorsed believable and valid problems more than unbelievable and invalid ones. Morsanyi and Handley (2012) used both simple and complex reasoning problems, however, other studies (e.g., Klauer \& Singmann, 2013; Singmann, Klauer, \& Kellen, 2014) failed to replicate this effect on complex arguments, indicating that the liking effect on complex problems was mainly from content of the arguments in Morsanyi and Handley's study rather than logical structures.

In later studies, the liking effect was replicated for simple reasoning arguments. For example, Trippas, Handley, Verde, and Morsanyi (2016) presented participants with a range of propositional argument types and asked them to rate their liking (Experiment 1) and evaluate the brightness (i.e., the font shade brightness) of the conclusion to each problem (Experiments $2 \& 3$ ). They found that participants judged logically valid and believable statements more likable and brighter than invalid and unbelievable statements. Moreover, a simulated smile (putting a pen between the lips; Experiment 3) affected the judgment of brightness which is consistent with the fluency-affect intuition model. These findings suggest that the logical structure of reasoning problems is readily accessible to intuitive processes.

\subsection{Individual Differences in Logical Intuitions}

The principal focus of the current paper is to examine the relationship between 'logical intuitions' and individual differences in cognitive capacity. Specifically, we aim to assess whether sensitivity to logical 
structure, across a range of judgment tasks, is independent of cognitive abilities, or varies as a function of cognitive ability in a similar way to the relationship often observed on explicit reasoning tasks (Newstead, Handley, Harley, Wright, \& Farrelly, 2004). This inquiry is related to a more general issue concerning the factors that differentiate good and bad reasoners - the divergence problem in reasoning and decision-making (De Neys \& Bonnefon, 2013). At what point, in the reasoning process, does the divergence between reasoners of different capabilities happen?

There is a substantial theoretical disagreement regarding the expected relationship between the output of $\mathrm{T} 1$ and $\mathrm{T} 2$ processes and cognitive abilities. On the one hand, some models propose that individual differences do not arise until the last stage of the reasoning process which is the point that reasoners engage in explicit, deliberative computation in the service of producing a response (Betsch \& Glöckner, 2010; Darlow \& Sloman, 2010; De Neys \& Bonnefon, 2013; Morsanyi \& Handley, 2012; Stanovich \& West, 2008; Toplak, West, \& Stanovich, 2014). On the other hand, according to other theorists, high and low ability reasoners diverge in their T1 thinking (Frey et al., 2017; Pennycook, Fugelsang, \& Koehler, 2012; Thompson \& Johnson, 2014; Thompson, Pennycook, Trippas, \& Evans, 2018) or even at the pre-reasoning stage (Mata, Ferreira, Voss, \& Kollei, 2017).

Regarding individual differences in $\mathrm{T} 1$ and $\mathrm{T} 2$ thinking, the default interventionist model generally assumes an asymmetrical correlation between the type of processes and the ability (cognitive ability) and willingness (cognitive style) to think analytically; T2 deliberative thinking depends on cognitive abilities whereas $\mathrm{T} 1$ processes are independent of working memory capacity, executive functions, and other cognitive abilities (Evans \& Stanovich, 2013; Stanovich, 1999; Stanovich \& West, 2008). As Evans (2008) has described, it seems that two separate systems control the behavior, the deliberative system with the dependence on executive functions and the intuitive one with independence from these resources. In a similar vein, Stanovich and West (2008) showed that performance in most of the tasks designed to examine biased thinking is independent of cognitive ability (CA). Based on these findings, these authors developed a 3-parameter model; mindware (storage), detection and override. According to this model, cognitive abilities are only able to differentiate reasoners in the override stage and they 
are not good predictors of variations in mindware and conflict detection. However, in his recent work, Evans (2017) has proposed that individual differences can also affect T1 thinking, as studies have shown that people with higher CA have different response patterns in tasks aimed to evaluate $\mathrm{T} 1$ thinking (Newstead et al., 2004; Thompson \& Johnson, 2014; Thompson et al., 2018).

Another way to investigate individual differences is to examine whether reasoners have different conflict detection abilities, and hence vary in logical intuition capacity. According to early models of logical intuition, since conflict detection is highly efficient and even the most biased reasoners can detect the conflict, bias arises as a consequences of the failure to override intuitive responses rather than lax monitoring (De Neys \& Bonnefon, 2013). The efficient conflict detection and the inefficient override imply that what determines the accuracy of the final response does not appear at the first stage of the reasoning. In other words, individual differences influence reasoners at the later stage of reasoning (inhibition) rather than in the early stages (storage or conflict detection; De Neys, 2015). This assumption is contradicted by a study of Pennycook et al. (2015) who showed, not only that the efficient conflict detection assumption is inaccurate, but also reasoners differ in their sensitivity to conflict signals. The most biased subset of the participants failed to detect the conflict between two opposing responses (see Experiment 1 and 2). Moreover, similar to previous studies (e.g., Pennycook et al., 2012), reasoners with a higher score in analytic cognitive style (ACS), a measure of willingness to engage in deliberative thinking, showed greater detection of the conflict. These findings imply that individual differences could influence both T1 and T2 processes. Following these findings and other evidence of early divergence (e.g., Mevel et al., 2015), a recent revision of the logical intuition model considers the possibility of conflict detection failure and hence an early divergence in the reasoning process (Frey et al., 2017). This conceptualization is supported by recent studies which found conflict detection efficiency is related to individual differences in cognitive abilities (Erceg, Galic, \& Bubić, 2019; Šrol \& De Neys, 2020).

It has been long established that people with higher cognitive abilities are more accurate in tasks that demand T2 processing (Toplak, West, \& Stanovich, 2011; Toplak et al., 2014). Since several 
models attribute some previously thought functions of $\mathrm{T} 2$ thinking to $\mathrm{T} 1$ processes, it is probable that some of the differences between high and low ability participants in reasoning problems arise from their differences in T1 thinking. Some support for this hypothesis comes from Thompson and colleagues' studies. For example, Thompson et al. (2018) used instructional manipulations along with different CA and ACS measures. The findings showed that, for participants with higher ability, logic-based responses were more accurate and more accessible than belief based-responses. This indicates that, for this group of reasoners, the automatic activation of the logical structure of the reasoning problem is the default process, even in the absence of $\mathrm{T} 2$ thinking. This pattern of findings was reversed for participants with lower abilities. Similarly, Thompson and Johnson (2014) demonstrated that even when participants were asked to respond as quickly as possible, those with higher CA and ACS more readily respond based on logical rules. In a recent study (Raoelison, Thompson, \& De Neys, 2020), researchers found that when participants were given another chance to rethink the responses that they gave whist under a memory load and limited time condition, the majority of correct responses were generated initially, rather than being corrected in the second stage. More importantly, cognitive abilities were more strongly associated with the tendency to generate the initial correct responses than with the tendency to correct the initial wrong responses.

These results, however, are inconsistent with Morsanyi and Handley (2012) who argued that intuitive sensitivity to logical validity is independent of cognitive abilities. They showed that liking ratings, as an index of $\mathrm{T} 1$ thinking, were not affected by instruction (Experiment 1), the figure of the syllogisms (Experiment 2), and participants' working memory capacity (Experiment 3). Moreover, a misattribution manipulation (i.e., background music) and affective priming (Experiment 4) influenced liking ratings but not validity judgments. Similarly, Nakamura and Kawaguchi (2016) did not find a significant correlation between thinking dispositions as measured by Rational-Experiential Inventory and liking judgments. This finding indicates that intuitive logic, like other $\mathrm{T} 1$ processes, is independent of cognitive capacity. Notably, however, these researchers also demonstrated that liking judgments were related to explicit reasoning; reasoners with better performance in the explicit reasoning task had higher liking ratings for valid problems. This finding was supported by Hayes and colleagues (Hayes, 
Wei, Dunn, \& Stephens, 2020) who demonstrated that differences in working memory predict both validity and liking judgments. These contradictory findings make it hard to extract any straightforward principle regarding the relationship between cognitive abilities and individual differences in intuitive logic.

\subsection{The Present Study}

As will be clear from the preceding discussion, there is increasing evidence from a number of paradigms that is consistent with the 'logical intuition' hypothesis. However, it is unclear whether the range of findings reported from these paradigms reflect operation of the same underlying constructs. We do not know, for example, whether sensitivity to logical validity on a liking judgment task is related to similar measures of sensitivity on a brightness judgment task; or to reduced confidence on conflict problems; or whether it is related to the increased interference of belief-logic conflict under instructions to respond based upon beliefs. It is also unclear what the relationship is between cognitive ability and individual differences in measures of logical intuition. As we have seen this is an important question, given that a number of theorists have argued that intuitive processes are independent of working memory and cognitive ability.

The present series of studies examine evidence for sensitivity to logical validity across several experimental tasks and reasoning problems, together with exploring the relationship between individual differences in logical sensitivity and variations in cognitive abilities. Our main objective is to determine whether 'logical intuitions' are stronger amongst participants of higher ability and show consistent individual differences across distinct experimental tasks.

To address this objective, we conducted four studies. In the first experiment, we used three different reasoning problems (syllogisms, conditionals, and disjunctions) as well as a cognitive ability (CA) measure. The first experiment consisted of a liking judgment task in which participants were instructed to rate their liking of the conclusion to each reasoning problem and a validity judgment task in which they were asked to judge the validity of the same conclusions. In the second experiment, we asked participants to judge the brightness of the conclusion to logical arguments (brightness judgments task) 
and then, in the second block, rate their liking of the conclusions of those arguments (liking judgments task). Liking judgment and brightness judgment tasks have been used in previous research as indices of logical intuition (Morsanyi \& Handley, 2012; Nakamura \& Kawaguchi, 2016; Trippas et al., 2016). Moreover, participants also completed a measure of analytic cognitive style (ACS) as well as a CA test. Whilst CA measures the ability to think analytically, ACS measures the willingness to engage in analytical thinking. In experiment 3, we investigated liking and brightness judgments under speeded and unspeeded conditions in order to test the relative engagement of deliberative thinking in liking and brightness judgments. Finally, in Experiment 4, participants were instructed to complete a brightness judgment task, a validity judgment task, and individual differences measures. This experiment serves as further evidence for the main claims of the study, whilst providing a replication of the previous experiments.

\section{Experiment 1}

Previous research has found that people like valid and believable conclusions to reasoning problems more than invalid and unbelievable ones and that this effect is independent of deliberative $\mathrm{T} 2$ thinking (Morsanyi \& Handley, 2012; Trippas et al., 2016; but see, Hayes et al., 2020). In this experiment, we used a similar liking judgment measure to examine intuitive sensitivity to logical structure. Participants were also instructed to judge the validity of the same problems in the second part of the experiment. We hypothesized that reasoners would rate valid and believable conclusions as more likable than their invalid and unbelievable counterparts. Moreover, to investigate individual differences in intuitive logic, participants completed a verbal and quantitative reasoning task designed to measure CA.

\subsection{Method}

\subsubsection{Participants}

Forty seven students from Macquarie University participated in this experiment (39 were female, $M$ age $=22.45, S D=6.96)$. Participants were fluent English speakers without any education in formal logic, and they received either two course credits or AU\$ 15 for their participation in the experiment. 


\subsubsection{Materials}

We used 72 reasoning problems which included 24 conditionals (12 modus ponens and 12 modus tollens), 24 disjunctions (12 affirmation inferences and 12 denial inferences), and 24 single-model syllogisms. Each participant was presented with a same number of problems in validity by believability cells (i.e., valid believable [VB], valid unbelievable [VU], invalid believable [IB], invalid unbelievable [IU]) for each subtype of conditionals and disjunctions. Syllogisms were selected such that there were 12 conclusions in an A-C direction (e.g., Some A are C) and 12 conclusions in a C-A direction (e.g., some C are A). Moreover, half of the conclusions contained the "some" quantifier (Some C are A) and the other half had the "no" quantifier (No A are C), balanced across each of the validity by believability conditions. Except for changing a few contents to culturally familiar ones for the Australian participants, all of the problem contents and structures were adapted from Trippas et al. (2016). An illustration of the materials can be found in Table 1.

Several studies have shown that the content rather the structure of problems affects participants liking ratings (Klauer \& Singmann, 2013; Singmann et al., 2014). In order to eliminate any confounding effect of content, we randomized the content across each logic by belief cell for each participant anew. For the conditionals, we created a set of 4 different problems with the same content but with different validity and believability status for each argument. Each problem was selected randomly from its related set. This randomization was constrained in a way such that each participant was presented with the same number of problems in each logic by belief cell for the total of 12 modus ponens and 12 modus tollens conditionals. This way, each participant was presented with a unique list of arguments with no association between validity and a problem's content. The same randomization procedure was performed for affirmations and denial disjunctions, with the additional randomization of the "p" and "q" order in the first premise to minimize any possible order effect of premise propositions ("either monkeys are primates, or they are rodents" vs. "either monkeys are rodents, or they are primates"). To randomly assign the contents to syllogistic structures, we combined 24 categories (e.g., marsupials) with their members (e.g., kangaroos) or non-members (e.g., parrots). For conclusions with the "no" quantifiers, believable conclusions were constructed by randomly pairing a category with its non- 
member (e.g., "no marsupials are parrots") and unbelievable conclusions were created by the combination of a category with one of its members (e.g., "no marsupials are kangaroos"). This pattern was reversed for conclusions featured with the "some" quantifiers. Combining a category with its member creates a believable conclusion ("some marsupials are kangaroos") and combining a category with its non-member results in an unbelievable conclusion ("some marsupials are parrots"). The middle "b" terms in the syllogistic structure are selected from a set of pseudowords (e.g., "firtes") to control for premise believability.

Table 1: Different problem types across validity and believability conditions that were used in experiments 1 to 4.

\begin{tabular}{|c|c|c|c|c|}
\hline \multirow{3}{*}{$\begin{array}{l}\text { Problem } \\
\text { Type }\end{array}$} & \multicolumn{2}{|c|}{ Valid } & \multicolumn{2}{|c|}{ Invalid } \\
\hline & \multirow[t]{2}{*}{ Believable } & \multirow[t]{2}{*}{ Unbelievable } & \multirow[t]{2}{*}{ Believable } & \multirow[t]{2}{*}{ Unbelievable } \\
\hline & & & & \\
\hline \multicolumn{5}{|l|}{ Conditionals } \\
\hline Modus ponens & $\begin{array}{l}\text { P1: If a bear catches a fish } \\
{[\mathrm{p}] \text { it is quick [q] }} \\
\text { P2: A bear catches a fish } \\
{[\mathrm{p}]} \\
\text { C: The bear is quick }[\mathrm{q}]\end{array}$ & $\begin{array}{l}\text { P1: If a bear catches a fish }[\mathrm{p}] \\
\text { it is slow }[\mathrm{q}] \\
\text { P2: A bear catches a fish [p] } \\
\text { C: The bear is slow [q] }\end{array}$ & $\begin{array}{l}\text { P1: If a bear catches a } \\
\text { fish [p] it is slow [q] } \\
\text { P2: A bear catches a fish } \\
\text { [p] } \\
\text { C: The bear is quick }[\neg q]\end{array}$ & $\begin{array}{l}\text { P1: If a bear catches a } \\
\text { fish }[\mathrm{p}] \text { it is quick }[\mathrm{q}] \\
\mathrm{P} 2: \mathrm{A} \text { bear catches a fish } \\
{[\mathrm{p}]} \\
\mathrm{C} \text { : The bear is slow }[\neg \mathrm{q}]\end{array}$ \\
\hline Modus tollens & $\begin{array}{l}\text { P1: If a bear fails to catch } \\
\text { a fish }[\mathrm{p}] \text { it is slow [q] } \\
\text { P2: A bear is quick }[\neg \mathrm{q}] \\
\text { C: The bear catches the } \\
\text { fish }[\neg \mathrm{p}]\end{array}$ & $\begin{array}{l}\text { P1: If a bear catches a fish }[p] \\
\text { it is slow }[q] \\
\text { P2: A bear is quick }[\neg q] \\
\text { C: The bear fails to catch the } \\
\text { fish }[\neg p]\end{array}$ & $\begin{array}{l}\text { P1: If a bear catches a } \\
\text { fish }[\mathrm{p}] \text { it is slow }[\mathrm{q}] \\
\text { P2: A bear is quick }[\neg q] \\
\text { C: The bear catches the } \\
\text { fish }[\mathrm{p}]\end{array}$ & $\begin{array}{l}\text { P1: If a bear fails to catch } \\
\text { a fish [p] it is slow [q] } \\
\text { P2: A bear is quick }[\neg \mathrm{q}] \\
\text { C: The bear fails to catch } \\
\text { the fish [p] }\end{array}$ \\
\hline \multicolumn{5}{|l|}{ Disjunctions } \\
\hline Affirmation & $\begin{array}{l}\text { P1: Either the sun is } \\
\text { yellow [p] or it is blue [q] } \\
\text { P2: The sun is yellow [p] } \\
\text { C: The sun is not blue } \\
{[\neg p]}\end{array}$ & $\begin{array}{l}\text { P1: Either the sun is yellow } \\
\text { [p] or it is blue [q] } \\
\text { P2: The sun is blue [q] } \\
\text { C: The sun is not yellow }[\neg p]\end{array}$ & $\begin{array}{l}\text { P1: Either the sun is } \\
\text { yellow [p] or it is blue [q] } \\
\mathrm{P} 2 \text { : The sun is blue [q] } \\
\text { C: The sun is yellow [p] }\end{array}$ & $\begin{array}{l}\mathrm{P} 1 \text { : Either the sun is } \\
\text { yellow [p] or it is blue [q] } \\
\mathrm{P} 2 \text { : The sun is yellow [p] } \\
\text { C: The sun is blue [q] }\end{array}$ \\
\hline Denial & $\begin{array}{l}\text { P1: Either the sun is } \\
\text { yellow [p] or it is blue }[\mathrm{q}] \\
\mathrm{P} 2 \text { : The sun is not blue } \\
{[\neg \mathrm{q}]} \\
\text { C: The sun is yellow }[\mathrm{p}]\end{array}$ & $\begin{array}{l}\text { P1: Either the sun is yellow } \\
\text { [p] or it is blue [q] } \\
\text { The sun is not yellow }[\neg p] \\
\text { The sun is blue }[q]\end{array}$ & $\begin{array}{l}\text { P1: Either the sun is } \\
\text { yellow [p] or it is blue [q] } \\
\text { The sun is not yellow } \\
{[\neg p]} \\
\text { The sun is not blue }[\neg q]\end{array}$ & $\begin{array}{l}\text { P1: Either the sun is } \\
\text { yellow [p] or it is blue [q] } \\
\text { The sun is not blue }[\neg q] \\
\text { The sun is not yellow } \\
{[\neg p]}\end{array}$ \\
\hline \multicolumn{5}{|l|}{ Syllogisms } \\
\hline $\begin{array}{l}\text { Single-Model } \\
\text { Syllogisms }\end{array}$ & $\begin{array}{l}\text { P1: No burtes are } \\
\text { marsupials } \\
\text { P2: All bees are burtes } \\
\text { C: No marsupials are bees }\end{array}$ & $\begin{array}{l}\text { P1: No burtes are } \\
\text { marsupials } \\
\text { P2: All kangaroos are burtes } \\
\text { C: No marsupials are } \\
\text { kangaroos }\end{array}$ & $\begin{array}{l}\text { P1: All burtes are } \\
\text { bees } \\
\text { P2: Some marsupials are } \\
\text { burtes } \\
\text { C: No marsupials are } \\
\text { bees }\end{array}$ & $\begin{array}{l}\text { P1: All burtes are } \\
\text { kangaroos } \\
\text { P2: All marsupials are } \\
\text { burtes } \\
\text { C: No marsupials are } \\
\text { kangaroos }\end{array}$ \\
\hline
\end{tabular}

Finally, reasoners' CA was measured with the first part of the AH4 general intelligence test (Heim, 1970). This part contains 65 verbal and mathematical reasoning questions $(\alpha=.88)$. Participants were given 10 minutes to answer as many questions as they could. Previous research has shown that this test 
can successfully predict performance across different reasoning tasks including syllogisms and conditionals (Newstead et al., 2004).

\subsubsection{Procedure}

The experiment had three separate blocks. In the first block, participants were asked to rate their liking of the problems' conclusion. We used a serial presentation format, according to which, the first premise was presented for 2.5 seconds and removed from the screen. Then, the second premise was shown for 2.5 seconds and vanished after this period. Finally, the conclusion was presented along with a question ("how much do you like this statement") and a rating scale from 1 (dislike it very much) to 6 (like it very much). Participants were instructed to rate their liking by relying on their intuitions and gutfeelings. In order to encourage participants to base their liking judgments on their gut feelings, there was no reference to logical reasoning in the instructions. The instructions were adapted from Trippas et al. (2016) and can be found in the supplementary materials.

In order to make sure participants have read all the components of each problem and not only the conclusion, after completing the liking judgments, participants performed a short memory recognition test that they were informed of at the beginning of the instructions. We asked participants to decide whether they had seen each of 20 words in the previous part. Half of the words were drawn from the problems presented during the liking judgment task and the other half were new. This memory test, which was also used following the brightness judgments in subsequent experiments, was designed to encourage participants to read all of the statements and not just the conclusion. Due to the sequential presentation of the arguments, some inattentive participants may approach the task by ignoring the first two premises and base their judgment on the conclusion alone. Participants who scored two standard deviations below the mean were excluded from the analysis of that specific task.

In the second block of the experiment, participants were given the same reasoning problems and were asked to judge the logical validity of each problem by clicking on an appropriate valid or invalid box. Since completing the validity task as the first task may have encouraged participants to base their subsequent liking judgments on an explicit evaluation of problem validity, there was no 
counterbalancing of the order of each block. However, to minimize the impact of liking judgments on subsequent validity judgments, we randomized content across logical structure again for this block. This way, we removed any systematic link between content and structure in both blocks of the experiment. Upon the completion of the liking and validity judgment parts, participants were presented with the CA test. They were instructed to solve 12 practice questions in an unlimited time and the actual test within 10 minutes.

\subsection{Results}

\subsubsection{Analysis Approach}

In all following experiments, we used the R programming language (R Core Team, 2014) for processing and analysing data, the afex package (Singmann et al., 2016) to perform mixed model analyses in experimental tasks, and the emmeans package (Lenth, Singmann, Love, Buerkner, \& Herve, 2018) for post-hoc analyses. Mixed model analysis is preferred to the common analysis of variance (ANOVA) because it takes all the fixed effects manipulated by researchers as well as the variability in participants and items into account (Baayen, Davidson, \& Bates, 2008). To set up each model, we started from the maximal model which is justified by the design (Barr, Levy, Scheepers, \& Tily, 2013) and, in cases where the full model is overparamaterized or degenerate, we simplified the random effect structure (Matuschek, Kliegl, Vasishth, Baayen, \& Bates, 2017). The simplification was performed by ommiting correlation parameters and higher-order effects from the random effect structure (Singmann \& Kellen, 2019). All post-hoc analyses were corrected for multiple comparisons using the Holm-Bonferroni adjustment. Conducting analysis of variance (ANOVA) for each mixed model, which can be found in the supplementary material, yields the same results.

In order to investigate individual differences in each experimental task, we also performed multiple regression analyses on logic effects in each task. Since different studies have shown that the traditional analysis of logic index by subtracting endorsement rates of valid arguments from invalid ones is somewhat flawed (Dube, Rotello, \& Heit, 2010; Heit \& Rotello, 2014), we used signal detection analysis (SDT) to compute logic effect indexes. The accuracy measures calculated by SDT fits the 
curved shape of reciever operating charactristics (ROCs) in reasoning literature (Dube et al., 2010). We used $d^{\prime}$ as the logic effect index for experiments 1 and 4 because the validity judgment task was responded to, using a binary response (valid and invalid) without any confidence ratings. It has been argued that $\mathrm{d}^{\prime}$ is a flawed measure of accuracy when the variances of signal and noise distributions are not equal (Heit \& Rotello, 2014; Rotello, Heit, \& Dubé, 2015). However, a recent meta-analysis of 22 reasoning studies demonstrated that equal variance SDT is still an appropriate model (Trippas et al., 2018). Thus, liking and brightness ratings in these two experiments were also converted to binary responses to reflect consistent logic effects. However, for experiments 2 and 3, we calculated $A_{z}$ as the more accepted measure of accuracy. We used the slope and intercept in the z-transformed hits against false alarms regressions for each participant to calculate $A_{z}$ (Macmillan \& Creelman, 2004). Again, performing regression analyses on the traditional logic indexes yields pretty consistent findings (see supplementary materials).

For the liking judgment, brightness judgment and individual differences tasks, participants with a memory score lower than two standard deviations from the mean of the total sample were excluded. The analysis of the validity judgment task was performed on the total sample. Analysing the whole sample did not change the main findings in each experiment. Moreover, one of the valid modus tollens items was actually invalid and we excluded this single item from further analyses. This item comprised less than $.01 \%$ of the whole data points. All data can be retrieved at the Open Science framework (https://osf.io/5dvcs/).

\subsubsection{Data Treatment}

Participants solved on average 43 problems correctly $(S D=8.25)$ in the AH4 test. Following from Trippas, Handley, and Verde (2013), we performed a median split on AH4 scores. Participants were divided into two groups of high and low CA based on the median score of 43 on this test. Following this procedure, the low CA group included 25 participants $(M=36.8, S D=4.85)$ and the high CA group comprised of 22 participants $(M=50, S D=5.16)$. For the liking judgment and individual differences parts, three participants with a low memory score were excluded. 


\subsubsection{Liking Judgments}

Liking rating means for each condition across the three problem types can be found in Table 2. A linear mixed model analysis was performed on liking rating scores. This model includes logic, belief, problem type and CA as fixed factors, by-subject random intercept, by-subject random slope for logic, belief, problem type and their 2-way interactions, by-item random intercept, and by-item random slope for logic, belief, and CA. The results revealed significant main effects of logic, $F(1,44.17)=48.77, p<$ .001 , belief, $F(1,47.22)=44.32, p<.001$, and problem type, $F(2,42.25)=10.36, p<.001$. These findings indicate that participants liked the conclusion of valid $(M=4.13)$ and believable $(M=3.92)$ problems more than the conclusions of invalid $(M=2.73)$ and unbelievable $(M=2.95)$ ones. Moreover, participants liked both conditionals $(M=3.60)$ and disjunctions $(M=3.51)$ more than syllogisms $(M=$ $3.19 ; p s<.002)$. There was no difference between liking ratings of disjunctions and conditionals $(p=$ $.28)$.

Table 2: Average liking and endorsement ratings (SDs) across cognitive ability groups for each tasks of Experiments 1

\begin{tabular}{llcclcc}
\hline & & \multicolumn{2}{c}{ Valid } & & \multicolumn{2}{c}{ Invalid } \\
\cline { 3 - 4 } \cline { 6 - 7 } & & Believable & Unbelievable & & Believable & Unbelievable \\
\hline \multirow{2}{*}{ Liking Judgments } & Low CA & $4.72(1.5)$ & $3.24(1.7)$ & & $3.57(1.7)$ & $2.40(1.4)$ \\
& High CA & $4.73(1.5)$ & $3.81(1.8)$ & & $2.79(1.6)$ & $2.17(1.4)$ \\
Validity Judgments & Low CA & $0.87(.33)$ & $0.80(.40)$ & & $0.26(.44)$ & $0.26(.44)$ \\
& High CA & $0.87(.33)$ & $0.84(.36)$ & & $0.10(.29)$ & $0.11(.31)$ \\
\hline
\end{tabular}

The findings showed a significant logic by belief interaction, $F(1,41.85)=6.38, p=.02$. Follow up analyses showed that the effect of logic was more pronounced for believable problems than unbelievable problems $(M$ differences $=1.54$ vs. 1.26). We also found significant interactions of logic by problem type, $F(2,39.98)=4.45, p=.02$, and belief by problem type, $F(2,25.87)=4.46, p=.02$. The results revealed that while the effect of logic and belief were significant across different problem types $(p s<.001)$, they were more pronounced on disjunctions. 

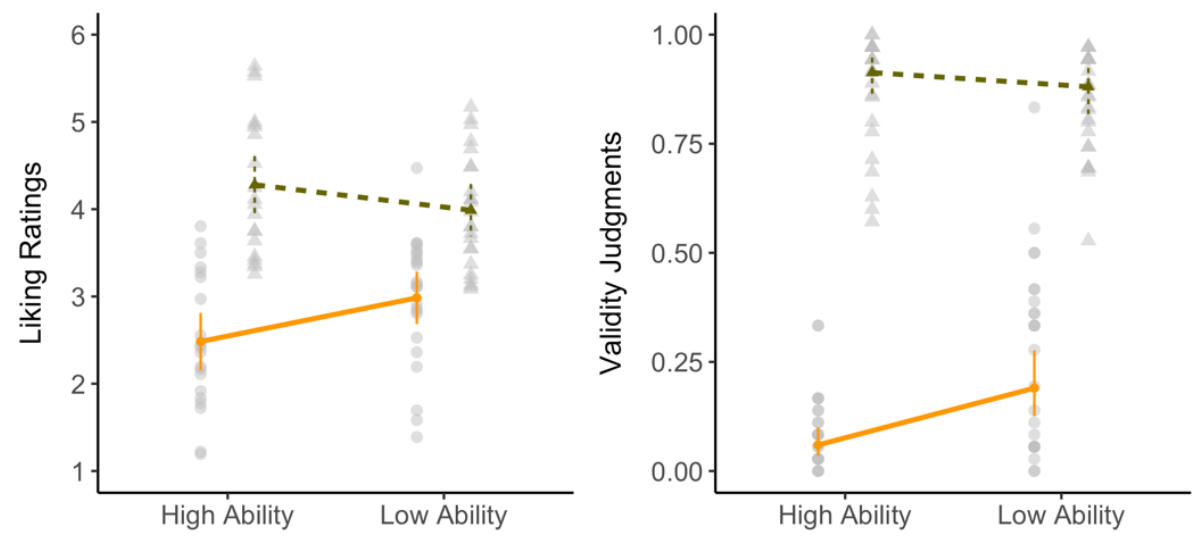

Validity $\rightarrow$ Invalid $\sim$ Valid

Figure 1: Liking and validity endorsement ratings of valid and invalid statements for high and low ability groups in Experiment 1. Error bars represent model-based standard error. Note that this variability measure does not allow for comparison between repeated-measure factors.

More importantly, the findings revealed a significant logic by CA interaction, $F(1,41.98)=4.03$, $p=.05$. The effect of validity was significant for both CA groups $(p s<.001)$, but as Figure 1 shows, the difference between liking rating for valid and invalid problems was larger for the high CA group compared to the low CA group ( $M$ differences $=1.8$ vs. 1$)$. There was also a significant belief by CA interaction, $F(1,41.97)=4.94, p=.03$. Although the effect of belief was significant for both groups ( $p s<.001)$, this effect was larger for the low CA group ( $M$ differences= 1.25 vs. .71).

\subsubsection{Validity Judgments}

The average endorsement rate for each ability group can be found in Table 2. A generalized linear mixed model with a log link and a binomial family distribution was performed on validity judgment scores. This model contains logic, belief, problem type, and CA as fixed factors, by-subject random intercept, by-subject random slope for logic, belief, and problem type, by-item random intercept, and by-item random slope for logic. No higher order interactions or correlation parameter were included in the random effect structure due to the convergence problem. The results revealed a significant main effect of logic, $\chi^{2}(1)=77.63, p<.001$, indicating that participants endorsed valid problems $(M=.90)$ more than invalid problems $(M=.11)$. The main effects of belief and problem type were not significant $\left(\chi^{2}=.86, p=.35\right.$, and $\chi^{2}=4.11, p=.13$, respectively) which is consistent with other findings in the 
literature, where belief effects are shown to be relatively weak on simple problems (Handley et al., 2011; Trippas et al., 2017). Participants endorsed believable problems $(M=.53)$ approximately at the same rate to unbelievable problems $(M=.49)$. The results showed a significant difference between total endorsement rates for the low CA group $(M=.57)$ compared to the high CA group $(M=.45), \chi^{2}(1)=$ $4.89, p=.03$.

The results revealed a logic by belief interaction, $\chi^{2}(1)=6.09, p=.01$, which, in line with the analysis of the liking judgments, showed that the effect of logic was more pronounced for believable problems $(M$ differences $=.81$ vs. .76). Moreover, logic by problem type interaction was marginally significant, $\chi^{2}(2)=6.09, p=.05$, which indicates that the effect of logic was more pronounced for disjunctions.

Similar to the liking judgment task, the results of the validity judgment task yielded a logic by CA interaction, $\chi^{2}(1)=6.85, p=.009$. As is apparent in Figure 1, whilst the effect of validity was significant for both ability groups $(p s<.001)$, it was larger for the high CA group compared to the low CA group $\left(M\right.$ differences $=.85$ vs. .69). There was no significant interaction between belief and CA $\left(\chi^{2}=.44, p=\right.$ $.51)$.

\subsubsection{Individual Differences}

The analyses in the previous section concerning individual differences were based on a common practice of the median split of a continuous variable (Iacobucci, Posavac, Kardes, Schneider, \& Popovich, 2015). In order to avoid the possible problems caused by artificial categorization of a variable (McClelland, Lynch, Irwin, Spiller, \& Fitzsimons, 2015; Rucker, McShane, \& Preacher, 2015), we performed two separate multiple linear regression analyses, one for the liking task and one for the validity task. In each regression model, the dependent variable was the logic effect in one task (e.g., the liking logic effect) and the predictors were CA and the logic effect in another task (e.g., the validity logic effect). As already explained, we used SDT indexes to calculate the logic effects for each task. 
Table 3: Multiple linear regression analyses predicting logic indexes with CA in both parts of Experiment 1

\begin{tabular}{llllllll}
\hline & Predictor & $\beta$ & $\mathrm{t}$ & $\mathrm{p}$ & $\mathrm{F}$ & $\mathrm{R}^{2}$ & $\mathrm{P}(\mathrm{F})$ \\
\hline \multirow{3}{*}{ Liking logic effect } & CA & .332 & 2.31 & .026 & & & \\
& Validity logic effect & .339 & 2.36 & .023 & & & \\
& & & & & 9.93 & .293 & .000 \\
Validity logic effect & .279 & 1.87 & .069 & & & \\
& LA & .352 & 2.36 & .023 & & & \\
& & & & & 8.70 & .264 & .000 \\
\hline
\end{tabular}

The findings revealed the liking logic effect was significantly correlated with both CA $(r=.48, p<$ $.001)$ and the validity logic effect $(r=.49, p<.001)$. Similarly, there was a significant correlation between the validity logic effect and CA $(r=.45, \mathrm{p}=.002)$. However, there was no significant difference in the magnitude of these correlations $(z=.23, p=.82,95 \% \mathrm{CI}[-.24, .30])$. As Table 3 shows, after entering $\mathrm{CA}$ and the liking logic effect simultaneously into a regression model, only the liking logic effect remains a significant predictor of the validity logic effect, although CA would comfortably reach significance as a predictor on a one-tailed test. On the liking judgment task, after controlling for the validity logic effect, a different pattern emerged, with CA and the validity logic effect emerging as significant independent predictors of the logic effect in the liking judgment task.

\subsection{Discussion}

To test the 'logical intuition' hypothesis, participants were asked to rate their liking of the conclusion to several reasoning problems. The results showed that subjects rated the conclusions to valid and believable arguments as more likable than those to invalid and unbelievable arguments. These results are consistent with the findings of experiments conducted by Morsanyi and Handley (2012), Trippas and colleagues (2016), and Nakamura and Kawaguchi (2016). According to these researchers, the underlying logical structure of the valid problems and its coherency create a sense of fluency. Despite being unaware of this conceptual fluency, reasoners find it desirable, which in result, leads to a positive affect. This affective product, then, will be used as a criterion in liking judgments (Topolinski, 2011; Topolinski \& Strack, 2009).

However, the results revealed that reasoners show individual differences regarding the logic effect in the liking judgment task. The interactions between logic and CA indicates that participants of higher 
cognitive ability show a greater effect of logical validity in their liking judgments compared to those with lower cognitive ability. Notably this finding is mirrored in the validity judgment data, where, consistent with numerous previous findings in the literature, participants with higher cognitive capacity show better logical performance (Stanovich \& West, 1997; Stupple, Ball, Evans, \& Kamal-Smith, 2011). The individual differences analysis confirms the alignment between liking and validity judgments. The logic effect for liking and the logic effect for validity are strongly correlated and predict one another. The regression models also show that cognitive ability independently predicts both logical validity and liking judgments to similar degrees. The most straightforward interpretation of the findings is that the liking judgment task is being approached, in part, as a reasoning task; that is participants are explicitly evaluating the validity of the conclusion and using this feature as a contributor to their judgments of liking. Those participants of higher ability are more able to do so accurately and hence show a greater effect of validity on liking judgments and higher accuracy on the equivalent reasoning task. This explanation is in line with Hayes and colleagues' (2020) study in which the logic effect in both liking and logic tasks were related to working memory capacity. Those authors concluded that the so-called "intuitive logic effect" in liking judgments is the result of explicit logical reasoning.

In the absence of any obvious and definitive features to base their liking judgments on, reasoners might evaluate the believability and validity of those statements as their criteria for their judgments. In other words, by engaging in deliberative thinking, it is possible that participants based their judgment on the validity of the statements instead of their own gut feelings or affective states. Considering these findings, one can argue that the deliberative components of liking judgment, and not the intuitive ones, renders such individual differences in judgments.

\section{Experiment 2}

The findings of Experiment 1 are consistent with previous research which has shown sensitivity to logical validity in liking judgments. Participants liked the conclusion to valid arguments significantly more than invalid arguments, despite being given clear instructions that the task required them to judge how much they liked the concluding statement based upon their 'gut instinct'. However, it is still unclear 
how the underlying structure influences liking ratings. As discussed above, one possibility is that participants engage in deliberative thinking while rating their liking and base their judgment explicitly on the logical validity of the argument. For example, Hayes et al. (2020) found that, participants with better working memory capacity and those under no working memory load have a greater logic effect in both liking and validity judgments.

For this reason, other researchers have used other measures of logical intuition. For example, Trippas et al. (2016) manipulated the contrast of the font of each problem and asked participants to judge the brightness of the final statement of a series of logical arguments. One could argue that on a brightness judgment task, participants are given an explicit instruction to respond to a problem feature that has no relationship to the outcome of an explicit reasoning process. In contrast to the liking ratings, in brightness ratings, there is an external criterion for right and wrong answers. A key question is whether the conceptual evaluation of logical validity will affect the perceptual evaluation of brightness despite the clear criterion for those perceptual judgments (Meier, Robinson, Crawford, \& Ahlvers, 2007). The rationale for this task is similar to the liking rating task. Coherency can lead to a positive affect which in turn is misattributed to the perceptual features of the conclusion leading to judgments of higher brightness in the same way it does with liking judgments. By using this method, researchers have found that reasoners judged valid problems as brighter than their invalid counterparts. In this experiment, we used the brightness judgment task as well as the liking judgment task.

Another issue regarding the first experiment is the individual differences measure. According to Stanovich (2018), intelligence tests, including the AH4 that we used in the previous experiment, are among optimal performance tasks. In these tasks, participants are asked to show their best performance and try to solve questions as accurately as they can. On the other hand, this is not similar to the realworld situations where there is not a signal for optimal responding. Normal performance tasks lack any cue to optimal performance. One example is the analytic cognitive style (ACS) task in which the structural features of the problem suggest a compelling and initial but wrong answer that needs to be overridden to reach a correct answer. As Stanovich (2018) proposed, conflict detection, as an index of 
logical intuition, may be more related to thinking disposition and style rather than CA. Thus, in order to examine individual differences in more depth, we included an ACS measure in the second experiment in addition to $\mathrm{CA}$.

Finally, Experiment 1 had a small sample size $(\mathrm{N}=47)$ which can result in an underpowered study. It has been argued that, depending on the effect size and the reliability of the measure, correlations can be extremely variable with small sample sizes (Schönbrodt \& Perugini, 2013). Moreover, with the reliability of $=.91$ for the liking judgment task and $=.88$ for the AH4 task, one needs at least 59 participants to find a correlation of .4 with $80 \%$ power (Parsons, Kruijt, \& Fox, 2019). Considering these reliability measures and a sample of 47 participants, the actual power of Experiment 1 was $70 \%$. Thus, the aim of Experiment 2 was to determine whether variations in logic effects for brightness judgments are related to similar variations on the liking judgment task using an experiment with a larger sample size.

\subsection{Method}

\subsubsection{Participants}

One hundred and forty five undergraduate students (120 were female, $M$ age $=22.86, S D=8.38$ ) from the Macquarie University completed the online version of the experiment. Participants were fluent English speakers and they took part in the experiment in exchange for two course credits.

\subsubsection{Materials}

In order to avoid any confounding effects, we randomly assigned the contrast value for each item, independent of its logical structure or content. To achieve this goal, we increased the number of reasoning materials to 96 problems; including 32 conditionals (16 modus ponens and 16 modus tollens), 32 disjunctions (16 affirmations and 16 denials), and 32 simple syllogisms. This way, we ended up with the same number of high and low contrast items in each validity by believability cell without disarranging the counterbalanced format of the reasoning materials in the first experiment. As an example, consider 16 modus ponens conditionals. For this subtype, we had 4 VB items, 4 VU items, 4 
IB items, and 4 IU items and for each of these conditions, half of the problems had high brightness values and the other half had low brightness values. This was the same for other problem types, including syllogisms. Moreover, to preserve the exclusivity of category and member pairs due to the addition of 8 new syllogisms, we created 16 categories each with 2 members. Besides these modifications, all the materials, structures, contents, and randomization methods were the same to Experiment 1.

We used the same manipulation and format for the physical brightness to those designed by Trippas et al. (2016). By changing the contrast of the black text against a white background, we created items with high and low brightness. High brightness items were created by randomly drawn an RGB (red, green, blue) value from a normal distribution with the mean of 30 and the standard deviation of 1 . Low brightness items were also constructed by randomly selecting a value from a normal distribution with the mean of 40 and the standard deviation of 1 . All elements of the problem, including premises, conclusion, response labels and the rating scale had the same RGB value.

Finally, to measure individual differences, we used the first part of the AH4 intelligence as an index of CA $(\alpha=.87)$ and, as an index of ACS, we used 6 incongruent base-rate problems taken from De Neys and Glumicic (2008), 3-item cognitive reflection test (CRT) from Frederick (2005), and 4-item CRT designed by Toplak et al. (2014). In the ACS task, which was presented before the AH4 task, participants were asked to respond to a series of questions as accurately as possible. All 13 problems, which can be found in the supplementary materials, were presented on a single screen in a random order. We counted the number of correct responses as the index of ACS. The fundamental feature of these items was cueing an intuitive but wrong answer upon the reading of the question (Pennycook, Cheyne, Barr, Koehler, \& Fugelsang, 2014; Trippas, Pennycook, Verde, \& Handley, 2015). To respond normatively, one needs to detect the wrongness of this initial answer and override it. This measure had strong reliability $(\alpha=.86)$.

\subsubsection{Procedure}


This experiment was designed in PsychoPy3 (Peirce et al., 2019), which has an acceptable timing precision (Bridges, Pitiot, MacAskill, \& Peirce, 2020), and was hosted on Pavlovia (https://pavlovia.org/). This experiment had a brightness judgment block, a liking judgment block, and an individual differences block. In the first block, participants were asked to judge the brightness of the conclusions to each reasoning problem on a scale from 1 (certainly low brightness) to 6 (certainly high brightness). The sequential presentation format and the timing of each premise was the same to the first experiment. After finishing the brightness task, participants performed a short memory recognition test in which they had to decide whether they had seen each of 20 words in the previous part.

In the second block, participants were given the same reasoning problems and were asked to rate their liking of each problem. At the end of this block, participants were presented with the same memory test. Since we assumed that the brightness judgment may provide a more theoretically-relevant measure of logical intuition compared to liking judgments, the order of those tasks was not counterbalanced. However, to minimize the order effect, we independently allocated content across logical structure for the second block. This way, we ended up with different lists of problems for the second block and removed any systematic link between content and structure in both blocks of the experiment. The presentation format for liking judgment task was the same to the first experiment. Upon the completion of the brightness judgment and liking judgment task, participants were presented with the ACS and CA measures. They solved 13 ACS questions first, in an unlimited time and then were instructed to solve 12 practice questions and 65 actual questions of the CA test.

\subsection{Results}

\subsubsection{Data Treatment}

The average of correct responses on the AH4 and ACS tests was $39.0(S D=9.30)$ and $5.39(\mathrm{SD}=3.66)$, respectively. Following the first experiment, we excluded 7 participants from the brightness judgment part and 8 participants from the liking judgment part of the analysis. These participants remain excluded for the individual differences analyses. We also performed a median split on CA and ACS measures. The median score for the CA measure was 40 which divided the sample into 67 high CA participants 
$(M=46.7, S D=5.16)$ and 78 low CA participants $(M=32.3, S D=6.55)$. Moreover, based on the median score of 5 on the ACS measure, two groups of 70 high ACS $(M=8.67, S D=1.97)$ and 75 low ACS $(M=$ 2.33, $S D=1.71)$ participants were created. High and low ACS are those with reflective and intuitive cognitive style, respectively (Trippas et al., 2015).

\subsubsection{Brightness Judgments}

The average brightness ratings for each cognitive ability group can be found in Table 4 . Since we had two theoretically separate measures of individual differences, we performed two separate linear mixed models for each of the CA and ACS measures. Both models successfully converged when by-subject random intercept, by-subject random slopes for logic, belief, brightness, and problem type, by-item random intercept, and by-item random slope for belief which explained the most variance, were included in the random effect structure.

The results for the first model showed significant main effects of logic $F(1,136)=17.51, p<$ .001 , belief, $F(1,140.37)=13.79, p<.001$, problem type, $F(2,65.77)=13.98, p<.001$, and brightness, $F(1,136.11)=48.80, p<.001$. Participants judged the conclusion to valid $(M=4.19)$, believable $(M$ $=4.21)$ and bright problems $(M=4.19)$ to be brighter than those to invalid $(M=3.97)$, unbelievable $(M$ $=3.96)$, and less bright $(M=3.97)$ problems. Participants also judged conditionals $(M=4.13)$ and disjunctions $(M=4.14)$ as brighter than syllogisms $(M=3.98$, both $p s<.001)$.

The results revealed significant logic by problem type, $F(2,12389.21)=3.73, p=.02$, and belief by problem type, $F(2,43.42)=6.14, p=.005$, interactions. Follow up analyses indicated that the logic effect was more pronounced on conditionals and disjunctions and the belief effect was more marked on disjunctions. The logic effect was significant on all problem types $(p s<.028)$. Whilst participants judged all believable syllogisms and disjunctions' conclusion as brighter than unbelievable ones ( $p s<$ $.001)$, the difference between believable and unbelievable conditional arguments was not significant ( $p$ $=.16$ ). Considering the relative complexity of syllogisms compared to disjunctions and modus tollens conditionals (Trippas et al., 2017), and the absence of a logical intuition effect on more complex problems observed in recent research (Brisson, Schaeken, Markovits, \& De Neys, 2018; Klauer \& 
Singmann, 2013; Singmann et al., 2014), it could be argued that brightness judgments were affected by task complexity. We also found a logic by belief by problem type by brightness interaction, $F(2$, $12414.67)=3.86, p=.02$. This interaction indicated that the three-way interaction of logic, belief, and problem type is more marked on high brightness arguments.

Table 4: Average brightness and liking rating (SDs) for each condition across cognitive ability groups in Experiment 2

\begin{tabular}{llccccc}
\hline & & \multicolumn{2}{c}{ Valid } & & \multicolumn{2}{c}{ Invalid } \\
\cline { 3 - 4 } \cline { 6 - 7 } & & Believable & Unbelievable & & Believable & Unbelievable \\
\hline \multirow{2}{*}{ Brightness Judgments } & Low CA & $4.39(1.4)$ & $4.04(1.6)$ & & $4.17(1.5)$ & $3.83(1.6)$ \\
\multirow{2}{*}{ Liking Judgments } & High CA & $4.25(1.4)$ & $4.08(1.5)$ & & $4.02(1.5)$ & $3.87(1.5)$ \\
& Low CA & $4.20(1.7)$ & $2.88(1.7)$ & & $3.83(1.7)$ & $2.65(1.5)$ \\
& High CA & $4.28(1.6)$ & $3.40(1.7)$ & & $3.82(1.7)$ & $2.95(1.6)$ \\
\hline
\end{tabular}

The findings revealed CA did not interact with belief, $F(1,136.02)=2.16, p=.14$, and logic, $F(1$, $135.94)=.02, p=.88$ (see Figure 2). Moreover, the results of the second model revealed that the logic by ACS interaction was not significant, $F(1,135.95)=.01, p=.90$. The key main effects and interactions in this model were similar to the previous model, except that we found a logic by ACS by problem type three-way interaction, $F(2,12403.86)=4.29, p=.01$. However, running separate models did not find a logic by ACS interaction across any problem types $(p>.19)$.
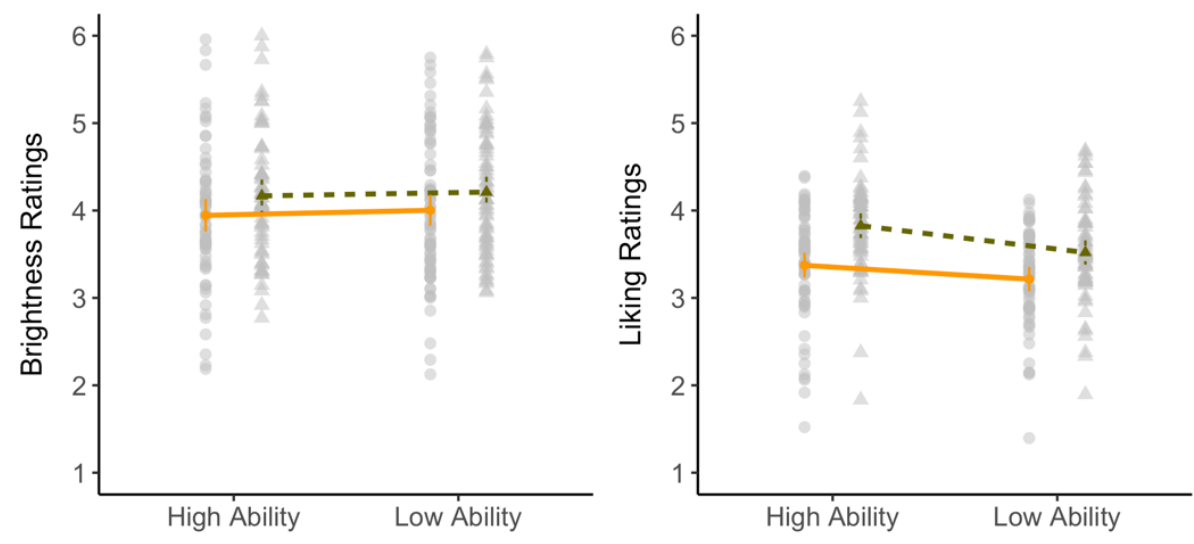

$$
\text { Validity } \approx \text { Invalid } \_ \text {Valid }
$$

Figure 2: Brightness and liking ratings of valid and invalid statements for high and low ability groups in Experiment 2. Error bars represent model-based standard error. 


\subsubsection{Liking Judgments}

The average liking ratings for each logic by belief cell can be found in Table 4. Following the analysis of brightness judgments, we conducted two linear mixed models on liking ratings. For the first model, we included logic, belief, problem type, and CA as fixed factors, by-subject random intercept, by-subject random slope for logic, belief, problem type and their two by two interactions, by-item random intercept, by-item random slope for logic, belief, CA, and their two by two interactions. For the second model, due to the convergence issue, we further simplified the model by removing ACS and two-way interactions from the by-item random structure. The results of the first model indicated that participants liked conclusions to valid problems $(M=3.67)$ more than those to invalid problems $(M=$ 3.29), $F(1,128.97)=38.73, p<.001$. They liked the conclusions of believable arguments $(M=3.96)$ more than the conclusions of unbelievable ones $(M=3), F(1,165.09)=76.72, p<.001$. The analysis revealed a significant main effect of problem type, $\mathrm{F}(2,74.60)=31.51, p<.001$. Participants liked both conditionals $(M=3.74)$ and disjunctions $(M=3.54)$ more than syllogisms $(\mathrm{M}=3.17$; ps <.001). They also rated conditionals as more likeable than disjunctions $(\mathrm{p}=.004)$.

We also found significant logic by problem type, $\mathrm{F}(2,50.48)=5.70, \mathrm{p}=.006$, and belief by problem type, $\mathrm{F}(2,81.28)=9.19, p<.001$, interactions. The effect of logic was more marked for conditionals, while the effect of belief was more pronounced for disjunctions. However, both the logic and belief effect were significant for all three problem types $(p s<.003)$. The logic by belief interaction was not significant $(F=1.92, p=.17)$, however this interaction was more marked for disjunctions, as indicated by a three-way interaction of logic by belief by problem type, $F(2,57.49)=6.31, p=.003$.

Finally, in contrast with the first experiment, the results showed a non-significant logic by CA interaction, $F(1,128.55)=1.48, p=.23$. Moreover, as the results of the second model demonstrated, ACS did not interact with logic, $F(1,134.91)=.44, p=.51$. On the other hand, belief interacted with both CA, $F(1,134.33)=5.13, p=.03$, and ACS, $F(1,134.96)=10.42, p=.002$. The belief effect was more marked for participants with low CA ( $M$ differences $=1.15$ vs. . 77$)$ and low ACS ( $M$ differences $=1.23$ vs. .70). 


\subsubsection{Individual Differences}

We calculated area under the curve $\left(A_{z}\right)$ in each task as the index of logic. The findings showed that the brightness logic effect was uncorrelated with CA $(r=.06, p=.49)$ or $\operatorname{ACS}(r=-.02, p=.84)$. However, the liking logic effect and the brightness logic effect had a significant relationship $(r=.48, p<.001)$. Moreover, both CA and ACS were non-significant predictors of the liking logic $(r=.13, p=.14$, and, $\mathrm{r}=.11, \mathrm{p}=.20$, respectively). The comparison of correlation analysis showed that there were no differences in the relationships of the brightness and liking logic effects with CA or ACS $(z=.80, p=$ $.43,95 \%$ CI $[-.24, .10]$, and $z=1.48, p=.14,95 \%$ CI $[-.04, .30]$, respectively).

Table 5: Multiple linear regression analyses predicting the logic indexes with CA and ACS in Experiment 2

\begin{tabular}{|c|c|c|c|c|c|c|c|}
\hline & Predictor & $\beta$ & $\mathrm{t}$ & $\mathrm{p}$ & $\mathrm{F}$ & $\mathrm{R}^{2}$ & $\mathrm{P}(\mathrm{F})$ \\
\hline \multirow{3}{*}{ Brightness logic effect } & $\mathrm{CA}$ & .020 & .248 & .805 & & & \\
\hline & ACS & -.077 & -.955 & .341 & & & \\
\hline & Liking logic effect & .483 & 6.20 & .000 & & & \\
\hline \multirow{5}{*}{ Liking logic effect } & & & & & 13.08 & .215 & .000 \\
\hline & $\mathrm{CA}$ & .071 & .890 & .375 & & & \\
\hline & $\mathrm{ACS}$ & .099 & 1.24 & .216 & & & \\
\hline & Brightness logic effect & .475 & 6.20 & .000 & & & \\
\hline & & & & & 14.08 & .229 & .000 \\
\hline
\end{tabular}

Including CA, ACS and the liking logic effect simultaneously in a regression model revealed that this model significantly predicted the logic effect in the brightness task. As Table 5 shows, the liking logic effect is a significant positive predictor of the logic effect in the brightness task, but this relationship is not explained by CA or ACS.

As is presented in Table 5, the brightness logic effect remained significant predictors of the liking effect even after entering all predictors simultaneously in a regression model. On the other hand, both CA and ACS were non-significant predictors of the liking effect.

\subsection{Discussion}

Experiment 2 used a novel brightness judgment task and replicated earlier findings in showing that participants' judgments concerning the perceptual qualities of a presented statement are significantly influenced by its logical status. Statements that follow from valid arguments are judged to be brighter 
than those that follow from invalid arguments. Interestingly, in the inferential analysis, there was no evidence that participants with higher cognitive ability or reflective style showed stronger effects of logic. The same results were found with liking judgments, where we failed to replicate the evidence from Experiment 1 which showed that participants of higher cognitive ability are more influenced by logical validity. This finding was confirmed by the regression analysis, where both CA and ACS were non-significant predictors of the liking logic effect and the brightness logic effect. Interestingly, liking and brightness logic indices were moderately correlated, a finding which suggests that there are some overlapping processes underpinning the logic effects across both tasks.

How might we explain the failure to replicate the liking logic and CA relationship observed in Experiment 1? One possibility, of course, is that the finding of a relationship in Experiment 1 was spurious. After all, the sample was small and the effect was relatively weak, whereas in Experiment 2 we recruited a larger sample, and included an additional measure of cognitive style. However, it is important to note that in Experiment 1 we did observe a robust relationship between CA and the logic effect under validity instructions and the relationship between the liking logic and the validity logic effects was strong. Perhaps liking judgments are influenced by both explicit deliberative reasoning processes and the automatic evaluation of structural coherency and depending on different cognitive top-down factors (i.e., cognitive ability) or contextual bottom-up factors (i.e., instruction or task complexity), both affect liking ratings to differing degrees. Having both components of intuitive and deliberative thinking may explain the discrepancy in the relationship between liking judgments and cognitive abilities in the current study and also in the literature (Hayes et al., 2020; Morsanyi \& Handley, 2012; Nakamura \& Kawaguchi, 2016). In Experiment 3, we further evaluated the role of deliberative processing in liking and brightness judgments using the speeded response paradigm.

\section{Experiment 3}

According to the speed assumption of the dual process (Evans, 2018), due to the effortless and automatic nature of intuitive processes, $\mathrm{T} 1$ thinking precedes $\mathrm{T} 2$ processes and provides an initial response. Thus, common practice to hinder $\mathrm{T} 2$ processes in the reasoning field is giving individuals a limited amount 
of time for responding. We used this manipulation in Experiment 3 on the assumption that limiting the response time minimizes any engagement in deliberative thinking. If we are correct in assuming that brightness judgments are mainly independent of deliberative processes, time pressure should not influence the logic effect in participants' ratings of physical brightness. Consistent with this hypothesis, Meier et al. (2007) found that the effect of conceptual evaluations on brightness judgments on a stimulus is relatively automatic and unintentional in a way that is not affected by a range of manipulations such as time pressure. On the other hand, if deliberative thinking is an active component in liking judgments, we would expect a reduced liking logic effect in the speeded task condition.

The design of this experiment had three major differences from the previous ones. First, in contrast to the second experiment, we manipulated only the contrast of the conclusion and used a black font for the two premises. Moreover, to test our hypothesis regarding the effect of time limitation, we introduced speed as a between subject factor. In other words, for each judgment task, we created a speeded and an unspeeded condition. Finally, in contrast to the Experiment 1 and 2, we counterbalanced the order of the brightness and liking tasks. This way we ended up with the 4 following groups, each with different speed and order conditions:

- Group 1: Speeded brightness judgments / Unspeeded liking judgments

- Group 2: Unspeeded brightness judgments / Speeded liking judgments

- Group 3: Speeded liking judgments / Unspeeded brightness judgments

- Group 4: Unspeeded liking judgments / Speeded brightness judgments

\subsection{Method}

\subsubsection{Participants}

One hundred and twenty undergraduate students (104 were female, $M$ age $=21.1, S D=6.37$ ) participated in the lab-based experiment. The recruitment procedure was the same to the previous experiments. Participants received either two course credits or AU\$ 15 in exchange of their time. 


\subsubsection{Materials}

Participants were presented with the same problems used in Experiment 2. We also administered the original three-item CRT $(\alpha=.67)$ and the AH4 test $(\alpha=.86)$ as measures of individual differences. All other problem structures and randomization procedures were the same to Experiment 2.

\subsubsection{Procedure}

In each group, participants were presented with a brightness judgment block (either speeded or unspeeded), a liking judgment block (either speeded or unspeeded), and an individual differences block. In order to operationalize the time pressure for the speeded tasks, we used the average reading time of arguments as a response deadline which was calculated based on a reading pre-test. This procedure has been successfully used in previous studies in order to minimize any engagement in explicit reasoning (e.g., Bago \& De Neys, 2017a, 2019). To that end, we performed a reading pre-test in which 8 participants were asked to read all of the 96 problems and to press the spacebar upon their reading of each statement. Results of the reading test showed that participants read the first premise in $1.62 \mathrm{~s}(S D$ $=0.42)$, the second premise in $0.9 \mathrm{~s}(S D=0.33)$ and the conclusion in $0.8 \mathrm{~s}(S D=0.23)$. We used the first two averages as the presentation time for the premises. However, since responding in the actual task required choosing the answer on a scale, which usually consumes more time compared to pressing a spacebar in the reading pre-test, we gave an extra 0.5 second for generating a response.

Moreover, in order to facilitate answering in such a limited time, we used a response scale of 1 (certainly low brightness/dislike it very much) to 4 (certainly high brightness/like it very much). Moreover, no response label and scale were presented to participants and they were asked to press the appropriate keyboard button. In trials where participant failed to respond in the allowed time, a feedback was provided requesting a faster response in the following trials. For the unspeeded tasks, the sequential presentation format and the timing of each statement was the same to the previous experiments. Finally, upon the completion of each task, participants performed a memory task and after the end of the second block, they answered to the individual differences' scales. 


\subsection{Results}

\subsubsection{Data Treatment}

In order to see if order affected judgments, we conducted two mixed models with logic, belief, order, and speed as fixed factors for brightness and liking tasks separately. We started with models containing random intercepts and slopes for all the fixed factors and perfomed the same simplification to the main analyses in case of convergence error. The results of these two models revealed that order did not interact with any of those factors $(p s>.05)$. Thus, we collapsed participants in different order groups into a single group. The exact formula and results of these two models can be found in the supplementary materials. Moreover, we did not include CA and ACS in brightness and liking judgments analyses to avoid a highly complex design. Thus, we entered speed as the only between-subject variable into the model. The average of correct responses to the AH4 was $42.1(S D=7.57) .53 \%$ of participants could not solve any of the CRT items correctly and the average mean of that scale was $.85(S D=1.05)$. The average score and the high percentage of zero correct answers have been found in other university student samples (Frederick, 2005). Participants with a memory score lower than 2 standard deviations from the average score were excluded. Consequently, 2 participants for the brightness judgment analyses and 7 participants for the liking judgment analyses were excluded.

Overall, participants failed to answer 402 items (3.4\%) in the speeded brightness tasks and 524 items $(4.5 \%)$ in the speeded liking tasks. Moreover, 4 participants in each task failed to answer more than 20 percent of items in the speeded conditions. However, by checking the pattern of their missing data, there was no systematic association between misses and validity, problem type, or believability. Thus, we did not exclude these participants. Missing data point are being handled in mixed models by using the partial pooling method, thus no replacement was performed on those values.

\subsubsection{Brightness Judgments}

The average ratings for each logic by belief cell can be found in Table 6 . A linear mixed model with logic, belief, problem type, brightness, and speed as fixed factors, by-subject random intercept, and bysubject adjustment to logic, belief, problem type, and brightness was conducted on brightness ratings. 
As expected, we found significant main effects of logic, $F(1,116.84)=14.41, p<.001$, belief, $F(1$, $116.82)=19.24, p<.001$, problem type, $F(2,112.35)=11.51, p<.001$, and brightness, $F(1,116.81)$ $=28.85, p<.001$. Participants rated valid $(M=2.51)$, believable $(M=2.53)$, and higher contrast $(M=$ $2.55)$ conclusions as brighter than invalid $(M=2.38)$, unbelievable $(M=2.36)$, and lower contrast $(M$ $=2.33)$ conclusions. Participants rated the conclusion to both conditionals $(M=2.52)$ and disjunctions $(M=2.44)$ to be brighter than syllogisms' conclusions $(M=2.37$; $p s<.007)$. They also judged conditionals as brighter than disjunctions $(p=.002)$.

Table 6: Average means of ratings (SDs) across two speed conditions for each tasks of Experiment 3

\begin{tabular}{lllllll}
\hline & & \multicolumn{2}{c}{ Valid } & & \multicolumn{2}{c}{ Invalid } \\
\cline { 3 - 4 } \cline { 6 - 7 } & & Believable & Unbelievable & & Believable & Unbelievable \\
\hline Brightness Judgments & Speeded & $2.56(.99)$ & $2.50(.97)$ & & $2.47(.98)$ & $2.44(.99)$ \\
& Unspeeded & $2.64(1.1)$ & $2.32(1.0)$ & & $2.44(1.1)$ & $2.18(1.0)$ \\
Liking Judgments & Speeded & $2.81(1.2)$ & $2.48(1.2)$ & & $2.63(1.2)$ & $2.25(1.2)$ \\
& Unspeeded & $3.20(1.1)$ & $2.23(1.2)$ & & $2.69(1.2)$ & $1.90(1.1)$
\end{tabular}

The results revealed that logic did not interact with speed $(F=1.64, p=.20)$. As hypothesized, the brightness logic effect was not influenced by time pressure manipulations. On the other hand, the findings indicated that belief interacted with speed, $F(1,116.82)=9.55, p=.002$. The belief effect was more pronounced in the unspeeded condition $(M$ differences $=.29$ vs. .05). Belief also interacted with problem type, $F(2,10177.65)=4.42, p=.01$. The effect of belief was more pronounced on disjunctions. This effect was more marked in the unspeeded task as is reflected in a three-way belief by problem type by speed interaction, $F(2,10177.65)=8.02, p<.001$. Finally, the brightness by problem type interaction indicated that the brightness effect was more pronounced for syllogisms and conditionals than disjunctions, $F(2,10175.45)=3.39, p=.03$.

\subsubsection{Liking Judgments}

A linear mixed model analysis was performed on liking rating scores. This model includes logic, belief, problem type and speed as fixed factors, by-subject random intercept, by-subject random slope for logic, belief, problem type, by-item random intercept, and by-item random slope for belief and speed. The results revealed significant main effects of logic, $F(1,111.55)=43.88, p<.001$, belief, $F(1,101.52)$ 
$=70.76, p<.001$, and problem type, $F(2,57.59)=27.36, p<.001$. Participants rated both valid $(M=$ 2.68) and believable $(M=2.81)$ conclusions as more likable than their invalid $(M=2.36)$ and unbelievable $(M=2.23)$ counterparts. They also rated the conclusion to both disjunctions $(M=2.51)$ and conditionals $(M=2.73)$ as more likable than syllogisms $(M=2.32 ; p s<.001)$. Moreover, conditionals were judged to be more likable than disjunctions $(p<.001)$.
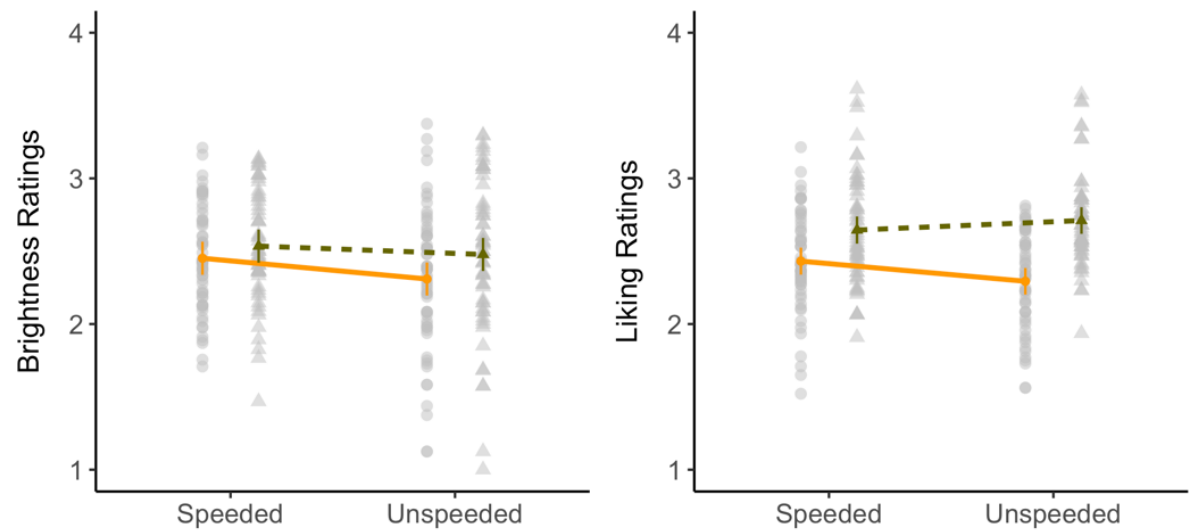

Validity $\approx$ Invalid $\leftarrow$ Valid

Figure 3: Brightness and liking ratings of valid and invalid statements for different speed groups in Experiment 3 Error bars represent model-based standard error.

The results revealed that logic interacted with speed, $F(1,111.50)=4.58, \mathrm{p}=.04$. As is shown in Figure 3, the logic effect was more marked in the unspeeded compared to the speeded condition ( $M$ differences $=.42$ vs. .21). Similarly, as shown in the brightness analysis above, belief interacted with speed, $F(1,111.80)=23.38, p<.001$. The belief effect was also more marked in the unspeeded condition $(M$ differences $=.84$ vs. .31$)$. Moreover, both logic and belief interacted with problem type, $F(2,9436.02)=9.88, p<.001$, and $F(2,34.48)=10.59, p<.001$, respectively. Although both effects were significant across different problem types $(p s<.001)$, the logic effect was more pronounced for conditionals and the belief effect was more pronounced for disjunctions. The belief by problem type interaction was more pronounced in the unspeeded task as shown by a 3 -way interaction, $F(2,9592.42)$ $=6.73, p<.001$. Finally, although the logic by belief interaction was not significant in general $(F=$ $1.76, p=.19)$, this interaction was more marked in the unspeeded task as is reflected in the three-way logic by belief by speed interaction, $F(1,9656.23)=7.42, p=.006$, and also it was more marked for 
disjunctions as is reflected in the three-way logic by belief by problem type interaction, $F(2,9564.72)$ $=4.24, p=.01$. In both of these analyses, logic was more pronounced for believable problems.

\subsubsection{Individual Differences}

Consistent with the second experiment, the brightness logic effect did not significantly correlate with either CA or ACS ( $r s=.08$ and .16 , respectively, $p s>.09$ ). On the other hand, the liking logic effect did significantly correlate with CA $(r=.22, p=.02)$, but not ACS $(r=.07, p=.5)$. Consistent with Experiment 2, comparing these correlations revealed that the differences in the relationships between the brightness and liking logic effects with CA and ACS were not statistically significant $(z=1.39, p=$ $.17,95 \% \mathrm{CI}[-.06, .34]$, and $z=.88, p=.38,95 \% \mathrm{CI}[-.11, .29]$, respectively). As in Experiments 1 and 2, we performed two separate multiple linear regression analyses, one for the brightness task and one for the liking task. As is presented in Table 7, both of these regression models were significant. For the brightness logic effect, the only significant predictor was the liking logic effect, consistent with the findings of experiment 2. In contrast, CA and the brightness logic effect were reliable predictors of logic in the liking task.

Table 7: Multiple linear regression analysis predicting logic indexes by CA and ACS in both tasks of the third experiment

\begin{tabular}{llcccccc}
\hline & Predictor & $\beta$ & $\mathrm{t}$ & $\mathrm{p}$ & $\mathrm{F}$ & $\mathrm{R}^{2}$ & $\mathrm{P}(\mathrm{F})$ \\
\hline \multirow{2}{*}{ Brightness logic effect } & CA & -.075 & -.77 & .441 & & & \\
& ACS & .158 & 1.68 & .096 & & & \\
& Liking logic effect & .412 & 4.60 & .000 & & & \\
& & & & & 8.15 & .163 & .000 \\
Liking logic effect & CA & .223 & 2.39 & .019 & & & \\
& ACS & -.083 & -.88 & .381 & & & \\
& Brightness logic effect & .401 & 4.69 & .000 & & & \\
& & & & & 9.30 & .184 & .000 \\
\hline
\end{tabular}

\subsection{Discussion}

Consistent with the findings of the previous studies, participants judged valid conclusions to be brighter and more likable than invalid conclusions. Although these effects were found to be significant in both speeded and unspeeded conditions, participants in the speeded task revealed a reduced liking logic effect. There was no evidence that speed attenuated the brightness logic effect. 
In contrast with Experiment 2 but consistent with Experiment 1, the individual differences analysis showed that cognitive ability predicted the liking but not the brightness logic index. As expected, the logic indices in the liking and brightness tasks were moderately correlated with one another suggesting overlapping processes. The distinct effect of time pressure and cognitive ability on liking and brightness judgments is consistent with the hypothesis that liking judgments involve some elements of deliberative, analytic processing. The intuitive component of this measure is also reflected in its correlation with logic effects in brightness judgments. The inconsistency in the relationship between the liking logic effect and cognitive abilities might be explained by the relative strength of each component in a specific task.

\section{Experiment 4}

In Experiment 1, we examined liking and validity judgments and showed a partial overlap between these two types of judgment in terms of relative effect of cognitive ability in participants ratings. In Experiments 2 and 3, we demonstrated that while the effect of logic on brightness and liking judgments may be influenced by a common factor, these two effects are not identical, with brightness judgments being less affected by the availability of cognitive resources and processing time. In Experiment 4, we aimed to investigate the relationship between validity and brightness judgments. If brightness judgments tend to better reflect the influence of logical intuition than liking judgments, there should be less of a relationship between brightness logic effects and with the validity logic effects than we observe with liking judgments. To examine this, we conducted an online experiment which provides us with an opportunity to test this hypothesis and replicate some of our previous findings with a large sample of participants.

\subsection{Method}

\subsubsection{Participants}

Two hundred undergraduate students $(183$ were female, $\mathrm{M}$ age $=22.2, \mathrm{SD}=6.78)$ completed the online version of the experiment. Participants received two course credits in exchange of their time. 


\subsubsection{Materials}

All reasoning problems and individual differences measures were the same to those that we used in Experiment 3. Both CA and ACS measures showed acceptable reliabilities $(\alpha=.91$ and $\alpha=.74$, respectively).

\subsubsection{Procedure}

Participants were instructed to respond to the two individual differences measures. Upon completing this block, they were presented with the brightness judgment task, the memory task, and the validity judgment task, respectively. The presentation format and timing were the same to previous unspeeded experiments. For the brightness judgment task, only the conclusion's contrast was manipulated and participants responded on a 6-point scale with the same contrast to the problem text. In the validity judgments task, participants provided their response on a binary response option.

\subsection{Results}

\subsubsection{Data Treatment}

The average accuracy scores were $39.5(S D=9.46)$ and $1.02(S D=1.14)$ for AH4 and CRT, respectively. Four participants were excluded from the analysis of the brightness judgments and individual differences due to the low memory score. We also divided participants into two groups of high CA $(M=47.3, S D=5.08)$ with 94 participants and low CA $(M=32.7, S D=6.77)$ with 106 participants, based on the median score of 40 on the AH4. Moreover, since median based split yielded two uneven ACS groups (135 vs. 65), we considered 95 participants with 0 correct answer as the low ACS group and 105 participants with 1 or more correct answers as the high ACS group $(M=1.94, S D$ $=.84)$. The results were similar when we used a median split.

\subsubsection{Brightness Judgments}

The average ratings for each condition across CA groups can be found in Table 8 . Two separate linear mixed models, one with CA and one with ACS as one of the fixed factors, were performed. For the first model, by-subject random intercept, by-subject random slope for all repeated-measure variables, by- 
item random intercept, and by-item random slope for belief and brightness were included. For the second model, by-item random intercept was excluded from the model due to the convergence problem. The results revealed main effects of logic, $F(1,194.22)=18.05, p<.001$, belief, $F(1,176.64)=21.57$, $p<.001$, problem type, $F(2,81.27)=21.99, p<.001$, and brightness, $F(1,128.71)=113.04, p<.001$. Participants judged the conclusion to valid $(M=3.64)$, believable $(M=3.64)$ and bright problems $(M$ $=3.70)$ to be brighter than those to invalid $(M=3.44)$, unbelievable $(M=3.44)$, and less bright $(M=$ 3.38) problems. Participants rated the conclusion to both conditionals $(M=3.60)$ and disjunctions $(M=$ 3.62) to be brighter than syllogisms' conclusions $(M=3.41 ; p s<.001)$. There was no difference in brightness ratings of conditionals and disjunctions' conclusions $(p=.55)$. Moreover, participants with lower CA and ACS had a higher overall brightness rating, $F(1,194.01)=12.13, p<.001$ and $F$ $(1,194.02)=4.17, p=.04$, respectively.

Table 8: Average brightness and endorsement ratings (SDs) for each condition across CA groups in Experiment 4

\begin{tabular}{llccccc}
\hline & & \multicolumn{2}{c}{ Valid } & & \multicolumn{2}{c}{ Invalid } \\
\cline { 3 - 4 } \cline { 6 - 7 } & & Believable & Unbelievable & & Believable & Unbelievable \\
\hline \multirow{2}{*}{ Brightness Judgments } & Low CA & $3.92(1.5)$ & $3.71(1.5)$ & & $3.77(1.5)$ & $3.56(1.5)$ \\
& High CA & $3.57(1.6)$ & $3.37(1.6)$ & & $3.31(1.6)$ & $3.14(1.6)$ \\
Validity Judgments & Low CA & $0.79(.41)$ & $0.71(.45)$ & & $0.37(.48)$ & $0.28(.45)$ \\
& High CA & $0.85(.35)$ & $0.80(.40)$ & & $0.22(.42)$ & $0.18(.38)$ \\
\hline
\end{tabular}

As expected, the logic by CA interaction was not significant, $F(1,194)=1.03, p=.31$, nor was the logic by ACS interaction, $F(1,194)=1.10, p=.30$. However, we found a weak three-way interaction of logic, CA, and problem type, $F(2,17633.28)=2.84, p=.06$. Although the logic by CA interaction was more marked on disjunctions, there was no evidence that this interaction was significant on any of those problem types. This finding was supported by running a similar model on each problem type separately. The logic by CA interaction was not significant in either of those models $(p s<.09)$. Finally, we found a significant logic by problem type interaction, $F(2,17604.07)=3.05, p=.05$, which indicated a larger effect of logic on conditionals. However, the logic effect was independently significant across all problem types $(p<.005)$.

\subsubsection{Validity Judgments}


For the validity judgment data, we performed two separate generalized linear mixed models with a log link and a binomial family distribution. In each model, either CA or ACS was included as a fixed factor. In the random structure, both models contain by-subject random intercept, and by-subject random slope for all repeated-measure effects. The first model also includes by-subject random effect correlation parameter. The results demonstrated main effects of logic $\chi^{2}(1)=246.5, p<.001$, and belief, $\chi^{2}(1)=$ 26.27, $p<.001$. Participants endorsed valid $(M=.83)$ and believable $(M=.58)$ arguments more frequently than invalid $(M=.21)$ and unbelievable $(M=.48)$ arguments. Participants with lower CA $(M=.55)$ tend to endorse more arguments than those with high CA $(M=.51), \chi^{2}(1)=5.88, p=.02$.

As Figure 4 shows, and consistent with Experiment 1, there was a significant logic by CA interaction, $\chi^{2}(1)=23.76, p<.001$. Logic also interacted with ACS, $\chi^{2}(1)=24.63, p<.001$. The effect of logic was more pronounced for groups with higher CA ( $M$ differences $=.71$ vs. .51$)$ and higher ACS ( $M$ differences $=.71$ vs. .49$)$. Follow up analyses revealed that whilst the logic by CA interaction was significant for all problem types $(\mathrm{p}<.003)$, it was more pronounced for conditionals, as was indicated by the logic, CA and problem type interaction, $\chi^{2}(2)=6.70, p=.04$. We also found a CA by problem type interaction, $\chi^{2}(2)=6.20, p=.04$, and a logic by problem type interaction, $\chi^{2}(2)=45.27, p<.001$. The difference between CA groups in terms of their endorsement ratings was more marked on conditionals. The logic effect, while significant for all problem types $(p s<.001)$, was also more marked on disjunctions.
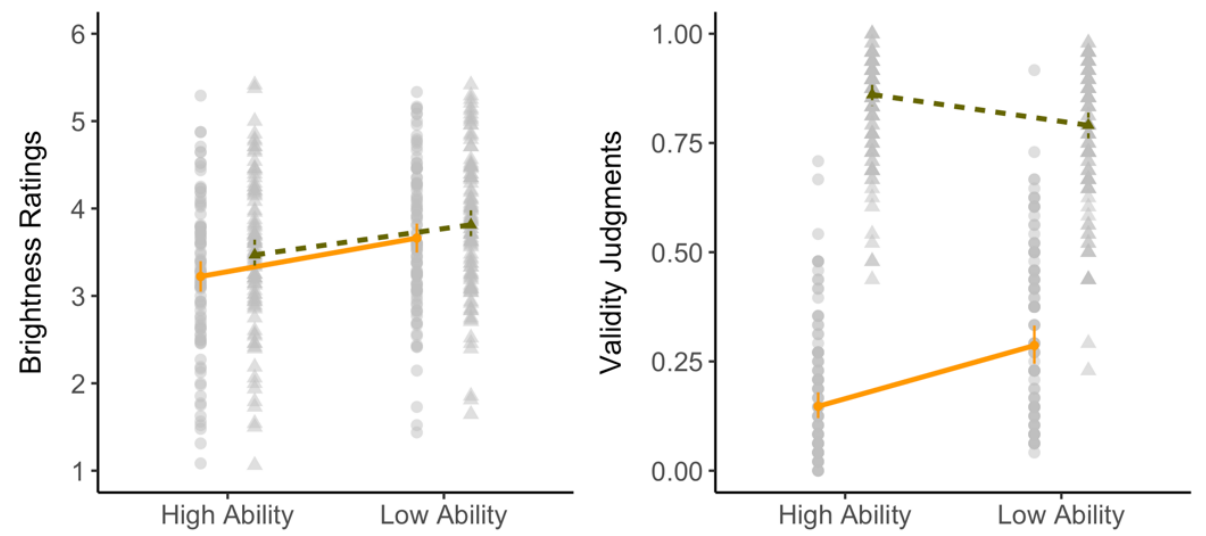

$$
\text { Validity } \approx \text { Invalid } \simeq \text { Valid }
$$




\subsubsection{Individual Differences}

The results revealed that the brightness logic effect did not significantly correlate with either CA, ACS, or the validity logic effect $(r s<.1, p s>.22)$. However, both CA $(r=.41, p<.001)$ and ACS $(r=.36$, $p<.001)$ were correlated with the validity logic effect. The results showed that the differences in the correlations between the brightness and validity logic effects with both CA and ACS were significant $(z=3.60, p<.001,95 \%$ CI $[.14, .49]$, and $z=4.92, p<.001,95 \%$ CI $[.26, .61]$, respectively). As is presented in Table 9, none of these variables yielded a significant model to predict the brightness logic effect. ACS was a marginally significant predictor, but in a negative direction. The validity logic effect, on the other hand, was significantly predicted by CA and ACS when all variables were entered simultaneously.

Table 9: Multiple linear regression analysis predicting logic indexes by CA and ACS in both tasks of Experiment 4

\begin{tabular}{llcccccc}
\hline & Predictor & $\beta$ & $\mathrm{t}$ & $\mathrm{p}$ & $\mathrm{F}$ & $\mathrm{R}^{2}$ & $\mathrm{P}(\mathrm{F})$ \\
\hline \multirow{3}{*}{ Brightness logic effect } & CA & .090 & 1.13 & .262 & & & \\
& ACS & -.152 & -1.95 & .053 & & & \\
& Validity logic effect & .110 & 1.35 & .179 & & & \\
& & & & & 2.01 & .015 & .115 \\
Validity logic effect & CA & .317 & 4.72 & .000 & & & \\
& ACS & .264 & 3.93 & .000 & & & \\
& Brightness logic effect & .086 & 1.35 & .179 & & & \\
& & & & & 19.32 & .220 & .000 \\
\hline
\end{tabular}

\subsection{Discussion}

The results of this experiment are consistent with minimal overlap between brightness judgments and validity judgments. Whilst participants with higher cognitive abilities showed a greater logic effect in their validity judgments, there was no relationship between cognitive ability and magnitude of the brightness logic effect. Moreover, the validity logic effect and the brightness logic effect were shown to be uncorrelated before and after controlling for cognitive resources. 


\subsection{Statistical Considerations}

The results of these experiments have revealed that the logic effect in validity judgments is consistently related to cognitive abilities, and the liking validity effect shows a relationship in some of our experiments but not all. Importantly, across all relevant studies, the logic effect in brightness judgments shows no relationship with cognitive ability, suggesting that sensitivity to logic in this condition may reflect intuitive logic that does not involve deliberative processing. However, the absence of a relationship can occur for many reasons, not least because the measurement in question has low variability or high measurement error. In this section, we considered two possible alternative statistical explanations for the absence of a relationship between these variables. We considered whether the nonsignificant correlation between CA and the brightness logic effect may be a result of a floor effect or measurement error, rather than reflecting the underlying true relationship between these variables.

According to the first alternative, a limited between-subject variability due to a floor effect in the brightness logic effect may lead to reduced correlations with other measures including cognitive ability. If the limited between-subject variability is responsible for the lack of relationship between the brightness logic effect and CA, one would expect to see a larger homogeneity and smaller standard deviation in the brightness logic effect compared to the liking logic effect. However, standard deviations for liking logic effects (SD Experiment $2=.11$, SD Experiment $3=.13$ ) were similar to those in the brightness logic effects (SD Experiment $2=.10$, SD Experiment $3=.11$ ) in experiments with both tasks. This amount of variability is evident in Figure 5, which plotted Az scores of each participant in the liking and brightness tasks of Experiments 2 and 3. As explained earlier, we have used the SDT sensitivity index of area under the curve (Az) as the measure of logic effect for each task. Az values of .5 , as is indicated by the vertical red line on the plots, reflect no differences in ratings of likability and brightness of valid and invalid arguments. Values below .5 reflect higher likability and brightness ratings for invalid arguments than valid ones and values above .5 indicate higher likability and brightness ratings for valid arguments than invalid ones. An inspection of Figure 5 suggests that, whilst most data points in both tasks fell around the vertical line, the variabilities are quite similar for both measures. 
Another possibility is that the smaller effect of logic in the brightness task compared to the liking task, and also the lack of relationship between the former and CA could be a result of low internal consistency and high measurement errors. The higher the measurement error, the lower between-subject variance, and the lower observed correlation between two variables (Parsons et al., 2019). This attenuation in correlation estimate is more obvious in studies, like our study, with an experimental task as a measure of individual differences (Hedge, Powell, \& Sumner, 2018). As is shown in Table 10, compared to the liking and validity logic effects, the split-half reliabilities for the brightness logic effects in all experiments are lower.
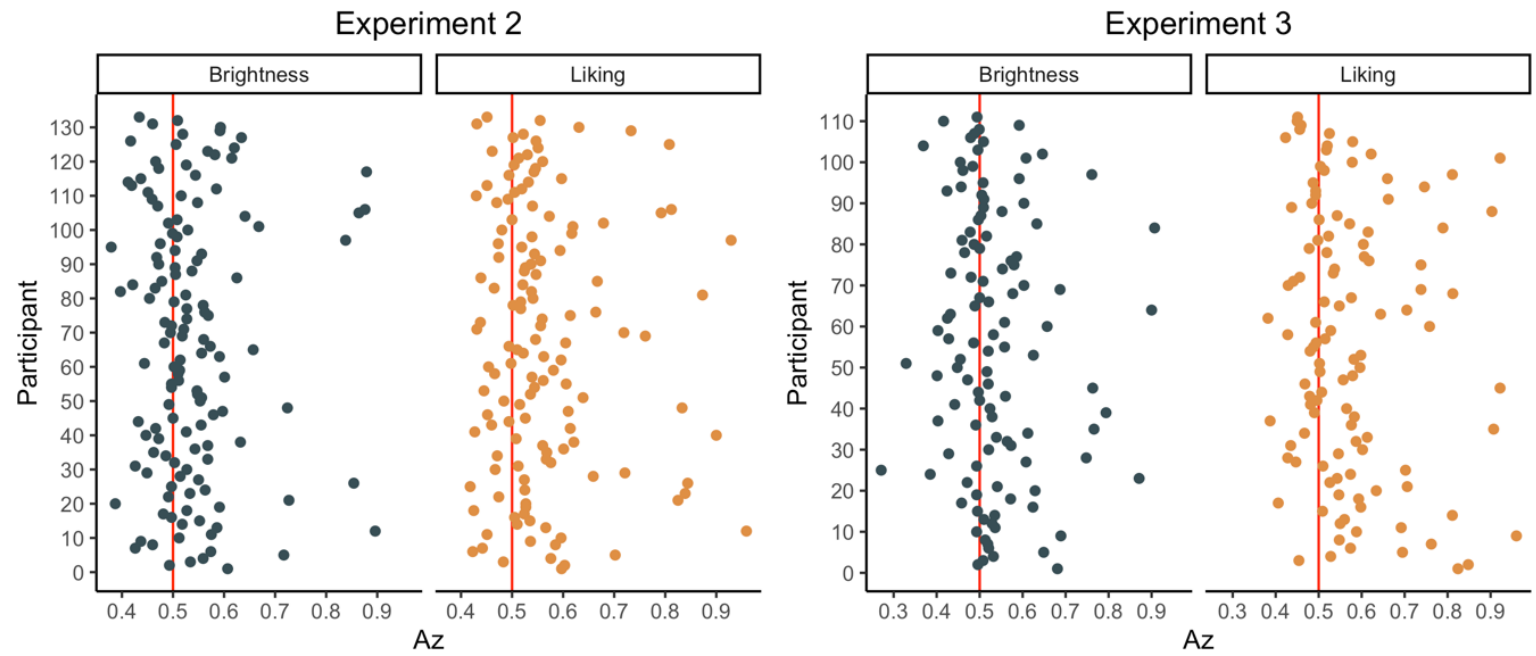

Figure 5: Area under the curve (Az) indexes for each participant in the brightness and liking tasks of experiments 2 (left panel) and 3 (right panel).

To correct for the attenuation in correlation between each logic effects (e.g., the brightness logic effect) and CA, we used Spearman's (1904) formula, which divides the observed correlation between two variables by the square root of the product of their reliabilities. We also reported bootstrapped confidence interval for dissattenuated estimates (Padilla \& Veprinsky, 2012). Finally, we performed two one-sided tests (TOST) to check whether dissattenuated estimates are still non-significant and practically close to zero. We specified $r_{L}=-.30$ and $r_{U}=.30$ as the upper and lower bounds of the smallest effect size of interest and conducted an equivalence test to see if the estimate falls within this boundary values (Lakens, Scheel, \& Isager, 2018). If the TOST showed to be significant, we can conclude that the dissattenuated estimate is practically equivalent to zero. 
As you can see in Table 10, even after dissattenuating correlations, the relationship between the brightness logic effects and CA in all experiments remained non-significant and practically equivalent to zero. Although the reliabilities of the brightness logic effects were relatively smaller than other effects, the attenuation of .03 indicated that the lack of relationship between this measure and CA may not be due to measurement errors.

Table 10: Reliability, attenuated and dissattenuated correlations with 95\% bootstrapped confidence intervals, and the equivalence tests of experimental effects of all experiments.

\begin{tabular}{cccccc}
\hline Experiment & Logic Effect & Task $\alpha$ & Observed r & Dissattenuated r & TOST (p-value) \\
\hline 1 & Liking & .91 & $.48^{* * *}$ & $.54[.31, .73]$ & .97 \\
& Validity & .86 & $.45^{* * *}$ & $.52[.27, .72]$ & .96 \\
2 & Brightness & .57 & .06 & $.08[-.14, .31]$ & .004 \\
& Liking & .71 & .13 & $.16[-.02, .36]$ & .046 \\
3 & Brightness & .62 & .08 & $.11[-.08, .31]$ & .019 \\
& Liking & .71 & $.22^{*}$ & $.28[.12, .46]$ & .41 \\
4 & Brightness & .59 & .09 & $.12[-.02, .26]$ & .004 \\
& Validity & .87 & $.41^{* * *}$ & $.46[.34, .56]$ & .99 \\
\hline
\end{tabular}

Although these analyses suggest that the lack of relationship between the brightness logic effect and CA might not be due to low variability, high measurement error, or low reliability, we would like to note that the results do not completely rule out these alternative explanations. Thus, future works can directly test these hypotheses. One possible method is to increase the between-subject variability and hence, the logic effect in the brightness task by minimising the gap between high and low brightness values. Decreasing the contrast between high and low brightness statements may increase the effect of logic on judgment since another source of judgment is diminished.

Finally, does the brightness logic effect appear because of a small subset of participants? To answer this question, we combined the data of Experiments 2 and $3(\mathrm{~N}=256)$ and calculated Az scores as the brightness logic effect. Then, we excluded participants with Az scores (or the brightness logic effect) greater than .8 and ran a random-intercept model with logic as the fixed factor. The logic effect remained significant $(F=41.85, p<.001)$. Then, we excluded those with $\mathrm{Az}>.7$ and again found a significant logic effect $(F=20.42, p<.001)$. Only after excluding a total of 43 participants with $\mathrm{Az}>.6$, did the logic effect became nonsignificant $(F=1.41, p=.236)$. This analysis shows that there is a distribution of brightness logic effects across our participant sample, but it is not the case that the effects 
arise only because of a small number of participants. Moreover, we calculated the brightness effect as the differences of ratings of high and low brightness items and found a very small non-significant negative relationship between the brightness logic effect and the brightness effect $(r=-.11, p=.067)$. A more detailed analysis of the brightness logic effect can be found in the supplementary materials.

\section{General Discussion}

According to some theoretical conceptualizations regarding how reason and intuition interact, access to the logical structure of problems requires primarily $\mathrm{T} 2$ thinking. This type of thinking is assumed to be responsible for monitoring the quality of the responses (Kahneman \& Frederick, 2002, but see, Evans, 2017) or correcting initial heuristic responses if required (Evans \& Stanovich, 2013). These frameworks have described $\mathrm{T} 1$ processes as a type of thinking which mainly operates on the superficial features of a problem (Kahneman \& Frederick, 2002; Sloman, 1996). The present studies aimed to investigate variations in people's ability to intuitively detect the validity of simple logical structures and to examine the extent to which reasoners with higher and lower cognitive capacities differ regarding this capability.

In four experiments, we used tests that have been claimed to measure intuitive sensitivity to logical structure across several reasoning problems, together with different individual differences measures to investigate both the existence of intuitive logic and individual differences in this capability. Our results showed that the conclusions to valid statements were rated as more likable and brighter than those to invalid statements. These liking and brightness effects for valid statements were replicated in Experiment 3 in which we used a limited response deadline and are consistent with recent empirical findings and theoretical conceptualizations (Hayes et al., 2020; Trippas et al., 2016). However, participants with higher cognitive ability (Experiments 1 and 3, but not Experiment 2) and unlimited processing time (Experiment 3 ) showed greater effects of logical validity in their liking judgments, a similar pattern to that shown with validity judgments in Experiments 1 and 4 . The logic effect in the brightness task, on the other hand, was unrelated to cognitive capacities. In other words, smarter and more reflective reasoners had no greater tendency to detect logical validity and translate this feature into their brightness judgments compared to their low capacity counterparts. 
How might we explain the pattern of findings across our four experiments? One possible hypothesis, which we call the continuum hypothesis of analytical engagement, is that the dependence on T1 and T2 processes to detect differences in logical structure varies on a continuum with certain tasks relying predominantly on explicit deliberative reasoning and others relying predominantly on implicit or intuitive processes. The validity judgment task is an apparently explicit reasoning task with a high chance of analytical thinking engagement, whilst liking and brightness judgments engage increasing degrees of intuitive processing with brightness judgment at the furthest end of the continuum. CA and ACS, as diagnostics of deliberative thinking, have a positive relationship with explicit reasoning on one side of the continuum. However, they are less reliable predictors of performance on the other side of the continuum as is reflected in the lack of relationship between individual differences measures and brightness judgments and the variable relationship between these measures and liking judgments. Logic effects arising from brightness judgments were also unrelated to validity judgments. Logic effects arising from liking judgments, lying in the middle of the continuum, have both intuitive and deliberative reasoning components. The deliberative components of liking judgments are reflected in the relationship between liking logic ratings, validity judgments, and CA. The intuitive component of this measure is also reflected in its correlation with logic effects in brightness judgments. Thus, according to this hypothesis, liking judgments overlap with both brightness and validity judgments. This continuum of analytical engagement is supported by the comparison of the correlation analyses which showed, whilst liking judgments and brightness judgments are not significantly different in terms of their reliance on cognitive abilities, there was a dissociation between brightness judgments and validity judgments as the former is independent of cognitive abilities.

In general, our findings suggest that individual differences in logical intuition, and more generally, in T1 thinking, are unrelated to cognitive capacities when any chance of engaging in deliberative thinking was hindered. In other words, participants with different cognitive ability and cognitive styles diverge, not at the beginning of the reasoning process, rather at the later point of this process. However, it should be noted that the current study investigated individual differences in respect to cognitive ability and styles. It could be the case that reasoners show an early divergence in respect to other cognitive 
factors, such as the degree of their attention to reasoning problems (Mata et al., 2017), the efficacy of their automatized logical rule (Stanovich, 2018), their metacognitive awareness of normative responses (Thompson \& Johnson, 2014), their level of conflict detection specificity (Bago, Raoelison, \& De Neys, 2019; Frey et al., 2017), or their reasoning strategies (de Chantal, Newman, Thompson, \& Markovits, 2019; Markovits, Brisson, de Chantal, \& Thompson, 2017).

Although our findings are not necessarily incompatible with the early divergence accounts, they are inconsistent with several studies which have shown that individual differences in cognitive abilities is related to intuitive logic. As we reviewed earlier, Thompson et al. (2018) found that, when participants were instructed to judge conclusions based on logical status, those with higher cognitive abilities had higher accuracy and lower response latencies. Similarly, Thompson and Johnson (2014) demonstrated that even when participants were asked to respond as quickly as possible, those with higher cognitive capacity are doing better to respond based on the logical rules. Moreover, conflict detection efficiency, as another index of intuitive logic, has been shown to be related to individual differences in cognitive abilities (Erceg et al., 2019; Šrol \& De Neys, 2020). Finally, cognitive ability is a better predictor of intuitively correct responses than deliberatively corrected responses (Raoelison et al., 2020).

Besides using different measures of logical intuition (with varying levels of complexity) and cognitive abilities (such as reasoning mindware and use of self-report measures of cognitive style) in the current study, there are several explanations that could account for this anomaly. First, one could argue that the relationship between fast logic and cognitive abilities reflects rapid engagement of T2 thinking. Given that reasoners are explicitly asked to respond based upon logic, perhaps reasoners of higher ability are simply more able to do so quickly. Thompson et al. (2018) tried to address this issue in their second experiment by applying a time pressure of 20 seconds, however, this amount of time still leaves the door open for engaging in deliberative thinking. As Bago and De Neys (2017a) found, the reading time for the long and short version of base-rate problems are approximately 12 seconds and 3 seconds, respectively, which are significantly lower than the amount of the time that participants had in Thompson et al.'s (2018) study. It is also worth noting that numerous psychometric studies have 
shown a strong relationship between intelligence and processing speed (see, for example, Vernon, 1983). It is quite possible that limiting the available time on a reasoning task allows participants of higher capacity and hence greater processing speed an advantage in performance which is then reflected in the observed covariance between cognitive ability and reasoning accuracy.

Second, as de Chantal et al. (2019) demonstrated, individual differences in reasoning strategies and attentional focus, compared to individual differences in working memory capacity, are more reliable predictors of reasoning performance on belief bias tasks. Reasoners with counterexamples strategies (e.g., attending more to premises and ignoring frequencies in favour of logic rules) showed higher accuracy and lower latency in deductive logic problems than those with statistical strategies. With the conflict detection paradigms, participants are explicitly instructed to respond based on logical necessity, which in turn, can result in a better performance for participants with more relevant strategies or logic mindware to apply those strategies, as mindware has been shown to be the best predictors of detection efficiency (Šrol \& De Neys, 2020). On the other hand, the tendency to engage in deliberative thinking on brightness judgments is assumed to be minimal and hence, the application of different reasoning strategies is, at the very least, unlikely. Future work could test this possibility through examining whether effects of logic on brightness judgments vary as a function of strategy use on explicit judgment tasks.

A key conclusion of our empirical work is that reasoners are intuitively sensitive to logical structure, but such sensitivity is reflected to differing degrees across distinct tasks. Recent dual process theorists have incorporated the existence of intuitive logic into their models of human reasoning (For a review, see, De Neys \& Pennycook, 2019; Thompson \& Newman, 2017). For example, in revising the default interventionist model, as one of the most influential dual process models, Evans $(2017,2018)$ suggested that reasoners are able to solve some simple reasoning problems through $\mathrm{T} 1$ processes. Moreover, Stanovich (2018) proposed a mindware continuum hypothesis, according to which, some logical rules which were practiced and automatized during development can cue normative responses without the engagement of T2 processes. Finally, in a revision of his model, Sloman (2012) attributed some levels 
of causal structure representation to intuitive and automatic processes ( see also, Hagmayer \& Sloman, 2009). On the other hand, several new dual process models, which have been proposed recently, address intuitive logic as one of the key components of their frameworks. These models include the parallel processing model (Handley \& Trippas, 2015; Trippas \& Handley, 2017), the logical intuition model or the hybrid model (De Neys, 2012, 2017), and the three-stage model of dual process theory (Pennycook et al., 2015), which commonly fall under the umbrella of the dual process theory 2.0 .

The parallel processing model (Handley \& Trippas, 2015; Trippas \& Handley, 2018) argues that there is not a one to one correspondence between problem features (e.g., believability or validity) and cognitive processing types (i.e., T1 and T2 thinking). This account claims that, depending on the complexity of the task at hand, $\mathrm{T} 1$ processes have access to the underlying logical structure of the problem and interestingly, on simple reasoning problems, the logical structure of a statement is more accessible than other features like its believability (Trippas et al., 2017). The fast logic hypothesis of this model is consistent with the observation of higher accuracy and lower speed for logic judgments (Handley et al., 2011; Pennycook, Trippas, et al., 2014) and the relative immunity of logic judgments from cognitive loading compared to belief judgments (Howarth et al., 2016; Howarth et al., 2018). Moreover, our observation of a reduced belief effect under the speeded task is consistent with the idea that there may well be a T2 component which explains some aspects of the influence of beliefs on cognitive judgments.

This fast logic is claimed to be an automatic and intuitive type of thinking which is produced upon the presentation of the problem as one of the many possible initial responses. In this regard, the parallel processing model is compatible with the three-stage model of analytical engagement (Pennycook et al., 2015) and the logical intuition model (De Neys, 2012, 2017a), according to which, at the onset of the reasoning process, several $\mathrm{T} 1$ responses are produced which can be overridden by $\mathrm{T} 2$ thinking in later stages of reasoning. One of these responses is a logical intuition and the absolute and relative strength of this response determines the final output of the reasoning process (De Neys \& Pennycook, 2019). Moreover, the early sensitivity to logic has been supported by electrophysiological evidence, especially 
by the early activation patterns of components which have attributed to the monitoring of conflict (Bago et al., 2018; Banks \& Hope, 2014). Altogether, these findings along with the current study results emphasize the need to revise the generic dual process theories regarding the characteristics of each processing type and their interaction. This need has been felt recently as is reflected in the recent revision of the dual process theory (Evans, 2017, 2018; Stanovich, 2018) and the development of the dual process theory 2.0 (De Neys, 2017b).

An important question is what are the underlying mechanisms that bring such intuitive judgments about? According to the fluency-affect intuition model (Topolinski, 2011, 2017), the ease with which a coherent argument is conceived produces a sense of fluency, and this fluency results in a positive affect. Although participants are aware of such an affective state, they have no clue regarding its source. Hence, this vague and ambiguous affect is misattributed to the judgments of liking (Reber \& Schwarz, 1999), truth (Topolinski \& Reber, 2010), and logical validity (Nakamura \& Kawaguchi, 2016). Accordingly, the conceptual fluency of coherent valid and believable arguments can produce a positive affect, which in turn may be misattributed to a judgment of physical brightness. Several theories argue that the coherency of information is among one of the mechanisms that logical intuition relies on (Betsch \& Glöckner, 2010; Glöckner \& Betsch, 2012; Topolinski, 2011).

Considering the coherency of information as a cue for judgment implies that reasoners are aware of the logical structure of the problem intuitively. This awareness reveals another proposed mechanism of logical intuition. According to the logical intuition model (De Neys, 2012) at least one heuristic intuitive response and one logical intuitive response is triggered upon the presentation of a reasoning problem. The final output of the reasoning process depends on the relative and absolute strength or activation level of these two responses. To test this account, Bago and De Neys (2019a) manipulated the strength of logical and heuristic intuition by changing the statistical or stereotypical information in the base-rate task. They found that not only the absolute strength of the two intuitions determined the proportion of normative or non-normative responses but also the relative strength of those intuitions affected the conflict detection rates. An interesting research question is whether manipulating the strength of logical 
intuition can influence the liking or brightness ratings of participants as observed on other intuitive measures?

Consistent with these hypotheses, most of the theories of "intuitive logic" propose logic as the underlying mechanism of logical intuition; a fact that is evident from the label for this concept. However, there is limited evidence to show that reasoners' judgments are genuinely impacted by the logical structure of the arguments rather than other structural features that happen to align with logical validity. It is quite possible that, instead of processing the true logical structure of problems, participants deploy a superficial heuristic. As one reviewer of this paper noted, in the case of conditionals, one possible heuristic is to match $\mathrm{p}$ and $\mathrm{q}$ and not-p and not-q. Whether participants have engaged in a true logical process or a superficial heuristic the responding pattern would be the same. Thus, the current findings cannot definitively support logic as the true underlying mechanism of intuitive logic over superficial heuristics. One way to directly test these two hypotheses would be to use indeterminate affirmation of the consequent (AC) arguments and determinate modus ponens (MP) arguments. Although all AC arguments are logically invalid, participants usually endorse one form (i.e., possible strong: pq; q; p) over the other (i.e., possible weak: pq; q; not-p; Evans, Handley, Harper, \& JohnsonLaird, 1999). Whilst a "true intuitive logic" account would predict logic effects (e.g., liking or brightness logic effects) only for MP arguments, a superficial heuristic account would predict a mirrored effect on both MP and AC arguments.

Last but not least, it should be noted that the current data do not necessarily support dual process theories over single process accounts. Recent studies have demonstrated that many of the findings claimed to provide support for dual process theories can also be explained by a single process account (Bonner \& Newell, 2010; Stephens, Dunn, \& Hayes, 2018). For example, Stephens et al. (2019) argued that inferring distinct cognitive processes based on dissociation in responding to different tasks in most of the dual process studies (including the current study) assumes a linear mapping from latent to observable dependent variables. By using the state-trace analysis which avoid such linearity assumption, these authors found that most of the dissociations in the reasoning tasks were disappeared. 
As another example, Bonner and Newell (2010) argued that longer response latencies in conflict detection studies could be interpreted by Hammond's cognitive continuum theory (Hammond, 1996). According to this theory, the cognitive processes vary on a single continuum from intuitive processes to deliberative ones and the characteristics of the task at hand determine the point at which the processing happens, with conflict problems shifting this processing to be more deliberative. One can note the similarity between this theory and the model that we proposed to explain our results. In both models, task features (e.g., instructional manipulations) determine the quality and/or quantity of deliberative thinking. A single process account could argue that explicit logic instructions induce reasoners to engage in deliberative processes, and liking and brightness instructions shift processes to the intuitive side, which is also less dependent on cognitive resources. Similarly, using the signed differences analysis, Hayes and colleagues (Hayes et al., 2020) showed that a parsimonious singleprocess signal detection model, according to which a single process underlies both liking and logic judgments, could explain the responding pattern in these tasks.

\subsection{Future Directions}

Since logical intuition is a relatively new concept in the realm of judgment and reasoning, there are few studies focusing on this capability and its cognitive mechanisms. Although several models in the dual process theory incorporate this concept as one of the main components of the reasoning process, more research is needed for a better understanding of logical intuition, and more generally, how reasoning and intuition interact. In the previous section, we proposed some possible studies to further explore logical intuition and replicate the current findings with different methods. For example, through examining the relationship between reasoning strategy and intuitive logic effects or by employing problems with different levels of determinacy or more abstract contents could broaden our knowledge of intuitive logic and its mechanisms.

Since investigating the conditions under which an unexpected effect disappears is a characteristic of the development of a program, as Kahneman and Frederick (2002) described, delineating the boundary conditions of logical intuition could be the next step in studying this phenomenon. Several 
studies have already addressed the boundary conditions of logical intuition in terms of argument complexity (Brisson et al., 2018; Frey et al., 2017; Klauer \& Singmann, 2013; Singmann et al., 2014). Individual differences can also provide a suitable opportunity to investigate the boundary conditions (Pennycook et al., 2012). By making a connection between individual differences studies and studies on the boundary conditions of logical intuition, we could investigate individual differences in different levels of complexity. For example, Klauer and Singmann (2013) found that reasoners have no intuitive sensitivity toward logic on more complex reasoning problem. However, it may be possible to find the logic effect on complex problems for high ability reasoners and by employing individual differences studies we may well develop a more precise picture of human reasoning abilities.

\subsection{Conclusion}

In four studies, we found that reasoners are intuitively sensitive to the logical validity of reasoning problems. However, different tasks showed different capacity to detect such intuitive sensitivity to logic. Brightness judgments, which rely predominantly on intuitive processes, did not vary as a function of available cognitive resources or processing time. On the other hand, these two factors affected liking judgments which indicates that liking ratings reflect both implicit and explicit reasoning ability. Moreover, the results revealed that reasoners with high and low cognitive abilities showed similar sensitivity to logic in tasks which minimised the requirement to engage in deliberative thinking. One implication of these results is that "[we] should have had more trust in the rationality of ... [our] homunculus", as Gigerenzer (2011) suggested. Generally, our results suggest a more optimistic view of human reasoning than the heuristics and biases literature (Kahneman \& Frederick, 2005) might suggest. We may be rational in principle but err in practice, where our beliefs continue to exert a powerful influence on our judgments. The findings suggest that, at least for simple arguments, the rudimentary logical machinery is effective and functioning. Hence a focus on decontextualising thinking may be an effective strategy to reduce reasoning biases as this would allow intuitive logic to be applied. There may also be scope in education to enhance logical intuition through intensive training in logical principles to enhance and accelerate the automatization of logical inference. Such an approach may be effective in countering the impact of bias in everyday thinking. 


\section{References}

Baayen, R. H., Davidson, D. J., \& Bates, D. M. (2008). Mixed-effects modeling with crossed random effects for subjects and items. Journal of Memory and Language.

https://doi.org/10.1016/j.jml.2007.12.005

Bago, B., \& De Neys, W. (2017a). Fast logic?: Examining the time course assumption of dual process theory. Cognition. https://doi.org/10.1016/j.cognition.2016.10.014

Bago, B., \& De Neys, W. (2017b). Rise and fall of conflicting intuitions during reasoning. Proceedings of the Annual Meeting of the Cognitive Science Society.

Bago, B., \& De Neys, W. (2019a). Advancing the specification of dual process models of higher cognition: a critical test of the hybrid model view. Thinking and Reasoning. https://doi.org/10.1080/13546783.2018.1552194

Bago, B., \& De Neys, W. (2019b). The Smart System 1: evidence for the intuitive nature of correct responding on the bat-and-ball problem. Thinking and Reasoning. https://doi.org/10.1080/13546783.2018.1507949

Bago, B., Frey, D., Vidal, J., Houdé, O., Borst, G., \& De Neys, W. (2018). Fast and slow thinking: Electrophysiological evidence for early conflict sensitivity. Neuropsychologia. https://doi.org/10.1016/j.neuropsychologia.2018.07.017

Bago, B., Raoelison, M., \& De Neys, W. (2019). Second-guess: Testing the specificity of error detection in the bat-and-ball problem. Acta Psychologica. https://doi.org/10.1016/j.actpsy.2019.01.008

Banks, A. P., \& Hope, C. (2014). Heuristic and analytic processes in reasoning: An event-related potential study of belief bias. Psychophysiology. https://doi.org/10.1111/psyp.12169

Barr, D. J., Levy, R., Scheepers, C., \& Tily, H. J. (2013). Random effects structure for confirmatory hypothesis testing: Keep it maximal. Journal of Memory and Language. https://doi.org/10.1016/j.jml.2012.11.001

Betsch, T., \& Glöckner, A. (2010). Intuition in judgment and decision making: Extensive thinking without effort. Psychological Inquiry. https://doi.org/10.1080/1047840X.2010.517737

Bonner, C., \& Newell, B. R. (2010). In conflict with ourselves? An investigation of heuristic and analytic processes in decision making. Memory and Cognition. https://doi.org/10.3758/MC.38.2.186

Bridges, D., Pitiot, A., MacAskill, M., \& Peirce, J. (2020). The timing mega-study: comparing a range of experiment generators, both lab-based and online. Retrieved from https://psyarxiv.com/d6nu5/download?format=pdf

Brisson, J., Schaeken, W., Markovits, H., \& Neys, W. De. (2018). Conflict detection and logical complexity. Psychologica Belgica. https://doi.org/10.5334/pb.448

Darlow, A. L., \& Sloman, S. A. (2010). Two systems of reasoning: Architecture and relation to emotion. Wiley Interdisciplinary Reviews: Cognitive Science. https://doi.org/10.1002/wcs.34

de Chantal, P. L., Newman, I. R., Thompson, V., \& Markovits, H. (2019). Who resists belief-biased inferences? The role of individual differences in reasoning strategies, working memory, and attentional focus. Memory and Cognition. https://doi.org/10.3758/s13421-019-00998-2

De Neys, W. (2012). Bias and conflict: A case for logical intuitions. Perspectives on Psychological Science, 7(1), 28-38. https://doi.org/10.1177/1745691611429354

De Neys, W. (2014). Conflict detection, dual processes, and logical intuitions: Some clarifications. Thinking and Reasoning, 20(2), 169-187. https://doi.org/10.1080/13546783.2013.854725

De Neys, W. (2015). Heuristic bias and conflict detection during thinking. Psychology of Learning and Motivation - Advances in Research and Theory. https://doi.org/10.1016/bs.plm.2014.09.001

De Neys, W. (2017a). Bias, conflict, and fast logic: Towards a hybrid dual process future? In Dual 
Process Theory 2.0. https://doi.org/10.4324/9781315204550

De Neys, W. (2017b). Dual process theory 2.0. Routledge. https://doi.org/10.4324/9781315204550

De Neys, W., \& Bonnefon, J.-F. (2013). The Whens and Whys of Individual Differences in individual thinking biases. Trends in Cognitive Sciences, 17(4), 172-178. https://doi.org/10.1016/j.tics.2013.02.001

De Neys, W., Cromheeke, S., \& Osman, M. (2011). Biased but in doubt: Conflict and decision confidence. PLoS ONE, 6(1). https://doi.org/10.1371/journal.pone.0015954

De Neys, W., \& Glumicic, T. (2008). Conflict monitoring in dual process theories of thinking. Cognition. https://doi.org/10.1016/j.cognition.2007.06.002

De Neys, W., Moyens, E., \& Ansteenwegen, D. V. (2010). Feeling we're biased: Autonomic arousal and reasoning conflict. Cognitive, Affective and Behavioral Neuroscience, 10(2), 208-216. https://doi.org/10.3758/CABN.10.2.208

De Neys, W., \& Pennycook, G. (2019). Logic, fast and slow: Advances in dual-process theorizing. Current Directions in Psychological Science.

De Neys, W., Vartanian, O., \& Goel, V. (2008). Smarter than we think: When our brains detect that we are biased. Psychological Science. https://doi.org/10.1111/j.1467-9280.2008.02113.x

Dube, C., Rotello, C. M., \& Heit, E. (2010). Assessing the belief bias effect with ROCs: It's a response bias effect. Psychological Review. https://doi.org/10.1037/a0019634

Epstein, S. (1994). Integration of the cognitive and the psychodynamic unconscious. American Psychologist. https://doi.org/10.1037/0003-066x.49.8.709

Erceg, N., Galic, Z., \& Bubić, A. (2019). Individual differences in abilities, knowledge and thinking dispositions among different types of problem solvers and their implications for the validity of reasoning tasks. Retrieved from https://psyarxiv.com/w5zau/

Evans, J., Handley, S. J., Harper, C. N. J., \& Johnson-Laird, P. N. (1999). Reasoning about necessity and possibility: A test of the mental model theory of deduction. Journal of Experimental Psychology: Learning, Memory, and Cognition. https://doi.org/10.1037/0278-7393.25.6.1495

Evans, J. S. B. T. (2008). Dual-Processing Accounts of Reasoning, Judgment, and Social Cognition. Annual Review of Psychology. https://doi.org/10.1146/annurev.psych.59.103006.093629

Evans, J. S. B. T. (2017). Dual process theory: Perspectives and problems. In Dual Process Theory 2.0. https://doi.org/10.4324/9781315204550

Evans, J. S. B. T. (2018). Dual-process theories. In International Handbook of Thinking and Reasoning (pp. 151-165). Routledge.

Evans, J. S. B. T., Handley, S. J., \& Bacon, A. M. (2009). Reasoning under time pressure: a study of causal conditional inference. Experimental Psychology, 56(2), 77-83. https://doi.org/10.1027/1618-3169.56.2.77

Evans, J. S. B. T., \& Stanovich, K. E. (2013). Dual-Process Theories of Higher Cognition: Advancing the Debate. Perspectives on Psychological Science. https://doi.org/10.1177/1745691612460685

Frederick, S. (2005). Cognitive reflection and decision making. Journal of Economic Perspectives. https://doi.org/10.1257/089533005775196732

Frey, D., Johnson, E. D., \& De Neys, W. (2017). Individual differences in conflict detection during reasoning. The Quarterly Journal of Experimental Psychology, 1-52. https://doi.org/10.1080/17470218.2017.1313283

Gigerenzer, G. (2011). Adaptive Thinking: Rationality in the Real World. Adaptive Thinking: Rationality in the Real World. https://doi.org/10.1093/acprof:oso/9780195153729.001.0001

Gigerenzer, G., \& Gaissmaier, W. (2010). Heuristic Decision Making. SSRN. https://doi.org/10.1146/annurev-psych-120709-145346 
Glöckner, A., \& Betsch, T. (2012). Decisions beyond boundaries: When more information is processed faster than less. Acta Psychologica. https://doi.org/10.1016/j.actpsy.2012.01.009

Gould, S. J. (1989). The Streak of Streaks. CHANCE. https://doi.org/10.1080/09332480.1989.10554932

Hagmayer, Y., \& Sloman, S. A. (2009). Decision Makers Conceive of Their Choices as Interventions. Journal of Experimental Psychology: General. https://doi.org/10.1037/a0014585

Hammond, K. (1996). Human judgment and social policy: Irreducible uncertainty, inevitable error, unavoidable injustice. Retrieved from https://books.google.com/books?hl=en\&lr=\&id=VxRnDAAAQBAJ\&oi=fnd\&pg=PR9\&dq=Ha mmond $+1996+$ Human + judgment + and + social + policy\&ots $=x v Q r K J K m 7 E \& s i g=f 4 c E f 4 \_F B R C 3$ OJMiuJ3IscPHK4k

Handley, S. J., Newstead, S. E., \& Trippas, D. (2011). Logic, Beliefs, and Instruction: A Test of the Default Interventionist Account of Belief Bias. Journal of Experimental Psychology: Learning Memory and Cognition, 37(1), 28-43. https://doi.org/10.1037/a0021098

Handley, S. J., \& Trippas, D. (2015). Dual processes and the interplay between knowledge and structure: A new parallel processing model. Psychology of Learning and Motivation - Advances in Research and Theory. https://doi.org/10.1016/bs.plm.2014.09.002

Hayes, B. K., Wei, P., Dunn, J. C., \& Stephens, R. G. (2020). Why is logic so likeable? A singleprocess account of argument evaluation with logic and liking judgments. Journal of Experimental Psychology: Learning, Memory, and Cognition. https://doi.org/10.1037/xlm0000753

Hedge, C., Powell, G., \& Sumner, P. (2018). The reliability paradox: Why robust cognitive tasks do not produce reliable individual differences. Behavior Research Methods. https://doi.org/10.3758/s13428-017-0935-1

Heim, A. (1970). AH 4 Group Test of General Intelligence. Manual. By A (lice) W (inifred) Heim. Rev. Ed.

Heit, E., \& Rotello, C. M. (2014). Traditional difference-score analyses of reasoning are flawed. Cognition. https://doi.org/10.1016/j.cognition.2013.12.003

Howarth, S., \& Handley, S. (2016). Belief bias, base rates and moral judgment: Re-evaluating the default interventionist dual process account. In The Thinking Mind: A Festschrift for Ken Manktelow. https://doi.org/10.4324/9781315676074

Howarth, S., Handley, S. J., \& Walsh, C. (2016). The logic-bias effect: The role of effortful processing in the resolution of belief-logic conflict. Memory and Cognition. https://doi.org/10.3758/s13421-015-0555-x

Howarth, S., Handley, S., \& Walsh, C. (2018). The logic sense: exploring the role of executive functioning in belief and logic-based judgments. Thinking \& Reasoning, 1-33. https://doi.org/10.1080/13546783.2018.1523808

Iacobucci, D., Posavac, S. S., Kardes, F. R., Schneider, M. J., \& Popovich, D. L. (2015). Toward a more nuanced understanding of the statistical properties of a median split. Journal of Consumer Psychology. https://doi.org/10.1016/j.jcps.2014.12.002

Kahneman, D., \& Frederick, S. (2002). Representativeness revisitet. Heuristics of Intuitive Judgment: Extensions and Applications. https://doi.org/10.1038/2251090a0

Kahneman, D., \& Frederick, S. (2005). A model of Heuristic Judgement. In The Cambridge handbook of thinking and reasoning. https://doi.org/10.1111//ogs.12119

Klauer, K. C., Musch, J., \& Naumer, B. (2000). On belief bias in syllogistic reasoning. Psychological Review. https://doi.org/10.1037/0033-295X.107.4.852

Klauer, K. C., \& Singmann, H. (2013). Does logic feel good? Testing for intuitive detection of logicality in syllogistic reasoning. Journal of Experimental Psychology: Learning Memory and 
Cognition, 39(4), 1265-1273. https://doi.org/10.1037/a0030530

Lakens, D., Scheel, A. M., \& Isager, P. M. (2018). Equivalence Testing for Psychological Research: A Tutorial. Advances in Methods and Practices in Psychological Science. https://doi.org/10.1177/2515245918770963

Lenth, R., Singmann, H., Love, J., Buerkner, P., \& Herve, M. (2018). Package 'emmeans.' R Package Version 1.15-15. https://doi.org/10.1080/00031305.1980.10483031>.License

Macmillan, N. A., \& Creelman, C. D. (2004). Detection Theory: A User's Guide: 2nd edition. Detection Theory: A User's Guide: 2nd edition. https://doi.org/10.4324/9781410611147

Markovits, H., Brisson, J., de Chantal, P. L., \& Thompson, V. A. (2017). Interactions between inferential strategies and belief bias. Memory and Cognition. https://doi.org/10.3758/s13421017-0723-2

Mata, A., Ferreira, M. B., Voss, A., \& Kollei, T. (2017). Seeing the conflict: an attentional account of reasoning errors. Psychonomic Bulletin and Review, 24(6), 1980-1986. https://doi.org/10.3758/s13423-017-1234-7

Matuschek, H., Kliegl, R., Vasishth, S., Baayen, H., \& Bates, D. (2017). Balancing Type I error and power in linear mixed models. Journal of Memory and Language. https://doi.org/10.1016/j.jml.2017.01.001

McClelland, G. H., Lynch, J. G., Irwin, J. R., Spiller, S. A., \& Fitzsimons, G. J. (2015). Median splits, Type II errors, and false-positive consumer psychology: Don't fight the power. Journal of Consumer Psychology. https://doi.org/10.1016/j.jcps.2015.05.006

Meier, B. P., Robinson, M. D., Crawford, L. E., \& Ahlvers, W. J. (2007). When "light" and "dark" thoughts become light and dark responses: Affect biases brightness judgments. Emotion. https://doi.org/10.1037/1528-3542.7.2.366

Melnikoff, D. E., \& Bargh, J. A. (2018). The Mythical Number Two. Trends in Cognitive Sciences. https://doi.org/10.1016/j.tics.2018.02.001

Mevel, K., Poirel, N., Rossi, S., Cassotti, M., Simon, G., Houdé, O., \& De Neys, W. (2015). Bias detection: Response confidence evidence for conflict sensitivity in the ratio bias task. Journal of Cognitive Psychology. https://doi.org/10.1080/20445911.2014.986487

Morewedge, C. K., \& Kahneman, D. (2010). Associative processes in intuitive judgment. Trends in Cognitive Sciences. https://doi.org/10.1016/j.tics.2010.07.004

Morsanyi, K., \& Handley, S. J. (2012). Logic feels so good-I like it! evidence for intuitive detection of logicality in syllogistic reasoning. Journal of Experimental Psychology: Learning Memory and Cognition. https://doi.org/10.1037/a0026099

Nakamura, H., \& Kawaguchi, J. (2016). People like logical truth: Testing the intuitive detection of logical value in basic propositions. PLoS ONE. https://doi.org/10.1371/journal.pone.0169166

Newman, I. R., Gibb, M., \& Thompson, V. A. (2017). Rule-based reasoning is fast and belief-based reasoning can be slow: Challenging current explanations of belief-bias and base-rate neglect. Journal of Experimental Psychology: Learning, Memory, and Cognition, 43(7), 1154-1170. https://doi.org/10.1037/xlm0000372

Newstead, S. E., Handley, S. J., Harley, C., Wright, H., \& Farrelly, D. (2004). Individual differences in deductive reasoning. The Quarterly Journal of Experimental Psychology. A, Human Experimental Psychology. https://doi.org/10.1080/02724980343000116

Newstead, S. E., Pollard, P., Evans, J. S. B. T., \& Allen, J. L. (1992). The source of belief bias effects in syllogistic reasoning. Cognition. https://doi.org/10.1016/0010-0277(92)90019-E

Padilla, M. A., \& Veprinsky, A. (2012). Correlation Attenuation Due to Measurement Error: A New Approach Using the Bootstrap Procedure. Educational and Psychological Measurement. https://doi.org/10.1177/0013164412443963

Parsons, S., Kruijt, A.-W., \& Fox, E. (2019). Psychological Science Needs a Standard Practice of 
Reporting the Reliability of Cognitive-Behavioral Measurements. Advances in Methods and Practices in Psychological Science. https://doi.org/10.1177/2515245919879695

Peirce, J., Gray, J. R., Simpson, S., MacAskill, M., Höchenberger, R., Sogo, H., ... Lindeløv, J. K. (2019). PsychoPy2: Experiments in behavior made easy. Behavior Research Methods. https://doi.org/10.3758/s13428-018-01193-y

Pennycook, G., Cheyne, J. A., Barr, N., Koehler, D. J., \& Fugelsang, J. A. (2014). Cognitive style and religiosity: The role of conflict detection. Memory and Cognition. https://doi.org/10.3758/s13421-013-0340-7

Pennycook, G., Fugelsang, J. A., \& Koehler, D. J. (2012). Are we good at detecting conflict during reasoning? Cognition. https://doi.org/10.1016/j.cognition.2012.04.004

Pennycook, G., Fugelsang, J. A., \& Koehler, D. J. (2015). What makes us think? A three-stage dualprocess model of analytic engagement. Cognitive Psychology. https://doi.org/10.1016/j.cogpsych.2015.05.001

Pennycook, G., Neys, W. De, Evans, J. S. B. T., Stanovich, K. E., \& Thompson, V. A. (2018). The Mythical Dual-Process Typology. Trends in Cognitive Sciences. https://doi.org/10.1016/j.tics.2018.04.008

Pennycook, G., Trippas, D., Handley, S. J., \& Thompson, V. A. (2014). Base rates: Both neglected and intuitive. Journal of Experimental Psychology: Learning Memory and Cognition, 40(2), 544-554. https://doi.org/10.1037/a0034887

R Core Team. (2014). R Core Team (2014). R: A language and environment for statistical computing. R Foundation for Statistical Computing, Vienna, Austria. URL Http://Www.R-Project.Org/.

Raoelison, M. T. S., Thompson, V. A., \& De Neys, W. (2020). The smart intuitor: Cognitive capacity predicts intuitive rather than deliberate thinking. Cognition. https://doi.org/10.1016/j.cognition.2020.104381

Reber, R., \& Schwarz, N. (1999). Effects of perceptual fluency on judgements of truth. Consciousness and Cognition, 8(1), 338-342.

Rotello, C. M., Heit, E., \& Dubé, C. (2015). When more data steer us wrong: replications with the wrong dependent measure perpetuate erroneous conclusions. Psychonomic Bulletin and Review. https://doi.org/10.3758/s13423-014-0759-2

Rucker, D. D., McShane, B. B., \& Preacher, K. J. (2015). A researcher's guide to regression, discretization, and median splits of continuous variables. Journal of Consumer Psychology. https://doi.org/10.1016/j.jcps.2015.04.004

Schönbrodt, F. D., \& Perugini, M. (2013). At what sample size do correlations stabilize? Journal of Research in Personality. https://doi.org/10.1016/j.jrp.2013.05.009

Singmann, H., Bolker, B., Westfall, J., Aust, F., Højsgaard, S., Fox, J., ... Love, J. (2016). afex: analysis of factorial experiments. $\mathrm{R}$ package version 0.16-1. R Package Version 0.16-1.

Singmann, Henrik, \& Kellen, D. (2019). An Introduction to Mixed Models for Experimental Psychology. In New Methods in Cognitive Psychology. https://doi.org/10.4324/97804293184052

Singmann, Henrik, Klauer, K. C., \& Kellen, D. (2014). Intuitive logic revisited: New data and a bayesian mixed model meta-analysis. PLoS ONE, 9(4). https://doi.org/10.1371/journal.pone.0094223

Sloman, S. A. (1996). The empirical case for two systems of reasoning. Psychological Bulletin. https://doi.org/10.1037/0033-2909.119.1.3

Sloman, S. A. (2012). Two Systems of Reasoning. In Heuristics and Biases. https://doi.org/10.1017/cbo9780511808098.024

Spearman, C. (1904). The Proof and Measurement of Association between Two Things. The American Journal of Psychology. https://doi.org/10.2307/1412159 
Šrol, J., \& De Neys, W. (2020). Predicting individual differences in conflict detection and bias susceptibility during reasoning. Thinking and Reasoning. https://doi.org/10.1080/13546783.2019.1708793

Stanovich, K.E. (1999). Who is rational? Studies in individual differences in reasoning. Studies of individual differences in reasoning.

Stanovich, Keith E. (2018). Miserliness in human cognition: the interaction of detection, override and mindware. Thinking \& Reasoning. https://doi.org/10.1080/13546783.2018.1459314

Stanovich, Keith E., \& West, R. F. (1997). Reasoning Independently of Prior Belief and Individual Differences in Actively Open-Minded Thinking. Journal of Educational Psychology. https://doi.org/10.1037/0022-0663.89.2.342

Stanovich, Keith E, \& West, R. F. (2008). On the Relative Independence of Thinking Biases and Cognitive Ability. Journal of Personality and Social Psychology. https://doi.org/10.1037/00223514.94.4.672

Stephens, R. G., Dunn, J. C., \& Hayes, B. K. (2018). Are there two processes in reasoning? The dimensionality of inductive and deductive inferences. Psychological Review. https://doi.org/10.1037/rev0000088

Stephens, R. G., Matzke, D., \& Hayes, B. K. (2019). Disappearing dissociations in experimental psychology: Using state-trace analysis to test for multiple processes. Journal of Mathematical Psychology. https://doi.org/10.1016/j.jmp.2018.11.003

Stupple, E. J. N., Ball, L. J., Evans, J. S. B. T., \& Kamal-Smith, E. (2011). When logic and belief collide: Individual differences in reasoning times support a selective processing model. Journal of Cognitive Psychology. https://doi.org/10.1080/20445911.2011.589381

Thompson, V. A., \& Johnson, S. C. (2014). Conflict, metacognition, and analytic thinking. Thinking and Reasoning. https://doi.org/10.1080/13546783.2013.869763

Thompson, V. A., \& Newman, I. R. (2017). Logical intuitions and other conundra for dual process theories. In Dual Process Theory 2.0. https://doi.org/10.4324/9781315204550

Thompson, V. A., Pennycook, G., Trippas, D., \& Evans, J. S. B. T. (2018). Do smart people have better intuitions? Journal of Experimental Psychology: General, 147(7), 945-961. https://doi.org/10.1037/xge0000457

Toplak, M. E., West, R. F., \& Stanovich, K. E. (2011). The Cognitive Reflection Test as a predictor of performance on heuristics-and-biases tasks. Memory and Cognition, 39(7), 1275-1289. https://doi.org/10.3758/s13421-011-0104-1

Toplak, M. E., West, R. F., \& Stanovich, K. E. (2014). Assessing miserly information processing: An expansion of the Cognitive Reflection Test. Thinking and Reasoning. https://doi.org/10.1080/13546783.2013.844729

Topolinski, S. (2011). A process model of intuition. European Review of Social Psychology. https://doi.org/10.1080/10463283.2011.640078

Topolinski, S. (2017). The sense of coherence: How intuition guides reasoning and thinking. In International Handbook of Thinking and Reasoning.

Topolinski, S., \& Reber, R. (2010). Immediate truth - Temporal contiguity between a cognitive problem and its solution determines experienced veracity of the solution. Cognition. https://doi.org/10.1016/j.cognition.2009.09.009

Topolinski, S., \& Strack, F. (2009). The analysis of intuition: Processing fluency and affect in judgements of semantic coherence. Cognition and Emotion. https://doi.org/10.1080/02699930802420745

Trippas, D., \& Handley, S. J. (2017). The parallel processing model of belief bias: Review and extensions. In Dual Process Theory 2.0. https://doi.org/10.4324/9781315204550

Trippas, D., \& Handley, S. J. (2018). The Parallel Processing Model of Belief Bias. In Dual Process 
Theory 2.0 (pp. 28-46). https://doi.org/10.4324/9781315204550-3

Trippas, D., Handley, S. J., \& Verde, M. F. (2013). The SDT model of belief bias: Complexity, time, and cognitive ability mediate the effects of believability. Journal of Experimental Psychology: Learning Memory and Cognition. https://doi.org/10.1037/a0032398

Trippas, D., Handley, S. J., Verde, M. F., \& Morsanyi, K. (2016). Logic brightens my day: Evidence for implicit sensitivity to logical validity. Journal of Experimental Psychology: Learning Memory and Cognition. https://doi.org/10.1037/xlm0000248

Trippas, D., Kellen, D., Singmann, H., Pennycook, G., Koehler, D. J., Fugelsang, J. A., \& Dubé, C. (2018). Characterizing belief bias in syllogistic reasoning: A hierarchical Bayesian metaanalysis of ROC data. Psychonomic Bulletin and Review. https://doi.org/10.3758/s13423-018$1460-7$

Trippas, D., Pennycook, G., Verde, M. F., \& Handley, S. J. (2015). Better but still biased: Analytic cognitive style and belief bias. Thinking and Reasoning. https://doi.org/10.1080/13546783.2015.1016450

Trippas, D., Thompson, V. A., \& Handley, S. J. (2017). When fast logic meets slow belief: Evidence for a parallel-processing model of belief bias. Memory and Cognition, 45(4), 539-552. https://doi.org/10.3758/s13421-016-0680-1

Tversky, A., \& Kahneman, D. (1983). Extensional versus intuitive reasoning: The conjunction fallacy in probability judgment. Psychological Review. https://doi.org/10.1037/0033-295X.90.4.293

Vernon, P. A. (1983). Speed of information processing and general intelligence. Intelligence. https://doi.org/10.1016/0160-2896(83)90006-5 